\title{
Conservative load transfer along curved fluid-solid interface with non-matching meshes
}

\author{
R.K. Jaiman ${ }^{a}$, X. Jiao ${ }^{b}$, P.H. Geubelle ${ }^{\mathrm{a}, *}$, E. Loth ${ }^{\mathrm{a}}$ \\ ${ }^{a}$ Department of Aerospace Engineering, University of Illinois, 306 Talbot Lab, 104 South Wright Street, Urbana, IL 61801, USA \\ ${ }^{\mathrm{b}}$ Computational Science and Engineering Division, Georgia Institute of Technology, Atlanta, GA 30332, USA
}

Received 29 August 2005; received in revised form 24 January 2006; accepted 16 February 2006

Available online 19 April 2006

\begin{abstract}
We investigate the effect of curvature on the accuracy of schemes used to transfer loads along the interface in coupled fluid-solid simulations involving non-matching meshes. We analyze two types of load transfer schemes for the coupled system: (a) point-to-element projection schemes and (b) common-refinement schemes. The accuracy of these schemes over the curved interface is assessed with the aid of static and transient problems. We show that the point-to-element projection schemes may yield inaccurate load transfer from the source fluid mesh to the target solid mesh, leading to a weak instability in the form of spurious oscillations and overshoots in the interface solution. The common-refinement scheme resolves this problem by providing an accurate transfer of discrete interface conditions across non-matching meshes. We show theoretically that the accurate transfer preserves the stability of the coupled system while maintaining the energy conservation over a reference interface. Finally, we introduce simple analytical error functions which correlate well with the numerical errors of the load transfer schemes.
\end{abstract}

(c) 2006 Elsevier Inc. All rights reserved.

Keywords: Coupled fluid-solid; Load transfer; Non-matching meshes; Point-to-element projection; Common refinement

\section{Introduction}

Non-overlapping domain decomposition methods with non-matching meshes have many applications ranging from contact mechanics [1-3], parallel processing [4,5] and electromagnetism [6]. The specific problem considered in this paper is the transfer of fluid traction field across dissimilar non-matching meshes in transient fluid-solid interaction (FSI). Such FSI problems are characterized by the dynamic nature of the interface loading and of the associated solid response, which may involve strong material (plasticity, visco-plasticity) and/or geometrical (large deformations and rotations) nonlinearity. The simulation of this class of problems is generally accomplished by loosely coupled schemes [7] based upon an explicit time marching scheme, with a Lagrangian finite element formulation for the solid domain, and an Arbitrary Lagrangian Eulerian (ALE)

\footnotetext{
* Corresponding author. Tel.: +1 217244 7648; fax: +1 2172440720.

E-mail address: geubelle@uiuc.edu (P.H. Geubelle).
} 
finite volume formulation for the fluid domain. The role of an FSI interface scheme is to ensure appropriate coupling between the two domains. This coupling procedure can be challenging when the two domains have non-matching meshes along the interface.

A number of interfacing methods have been proposed to solve FSI problems and can be categorized as either non-conservative or conservative schemes. The former do not conserve the work done by the fluid tractions transferred to the solid mesh, and therefore need fine meshes for both the fluid and solid domains to give reasonable results [8,9]. Traditional conservative methods rely on point-to-element transfer, e.g., nodeprojection [10] and quadrature-projection [11] schemes, and on point-to-point transfer [12]. However, these conservative methods often suffer from a lack of accuracy in transferring loads across non-matching meshes as presented for the point-to-element schemes in [13].

An alternative approach described in $[13,14]$ utilizes a least squares projection to achieve both accuracy and conservation of energy. This approach includes an orthogonal projection of two non-matching meshes and a systematic subdivision of the surface of intersection into "subelements" by constructing a common refinement of the input meshes. This subelement-to-subelement transfer enables robust and accurate numerical quadrature scheme to evaluate the spatial integral defining the load transfer.

In a recent paper [13], we have systematically assessed the accuracy of the point-to-element and subelementto-subelement load transfer schemes for flat non-matching interfaces. In the point-to-element projection schemes, the integrand exhibits discontinuities that generally lead to load transfer errors depending on the fluid-solid grid mismatch along the interface. These errors not only affect the solution along the interface but also can significantly undermine the solutions in the fluid and solid subdomains. In addition, the spurious oscillations and overshoots along the interface may cause a weak instability in the FSI simulations in terms of spurious oscillations and overshoots [15].

On the other hand, we showed in [13] that, by utilizing the common-refinement based mapping and integrating over the subelements, the error in the subelement-to-subelement transfer becomes independent of the element mismatch along the interface. As a result, the solution obtained with non-matching meshes is effectively identical to the solution associated with matching meshes. These findings were demonstrated first with the aid of a simple 1D static load transfer analysis, and then through three transient FSI problems of increasing complexity.

The studies of load transfer schemes presented in [13] assumed the interfaces to be flat and the geometries of the interface meshes to match well. For practical problems, the interface between fluid and solid domains are often curved, and hence the geometries of the fluid and solid meshes may mismatch severely along the interface (Fig. 1), although they approximate the same physical interface. This mismatch can lead to discrepancies in the normals, areas, etc., and hence affect adversely the accuracy of the coupled simulations.

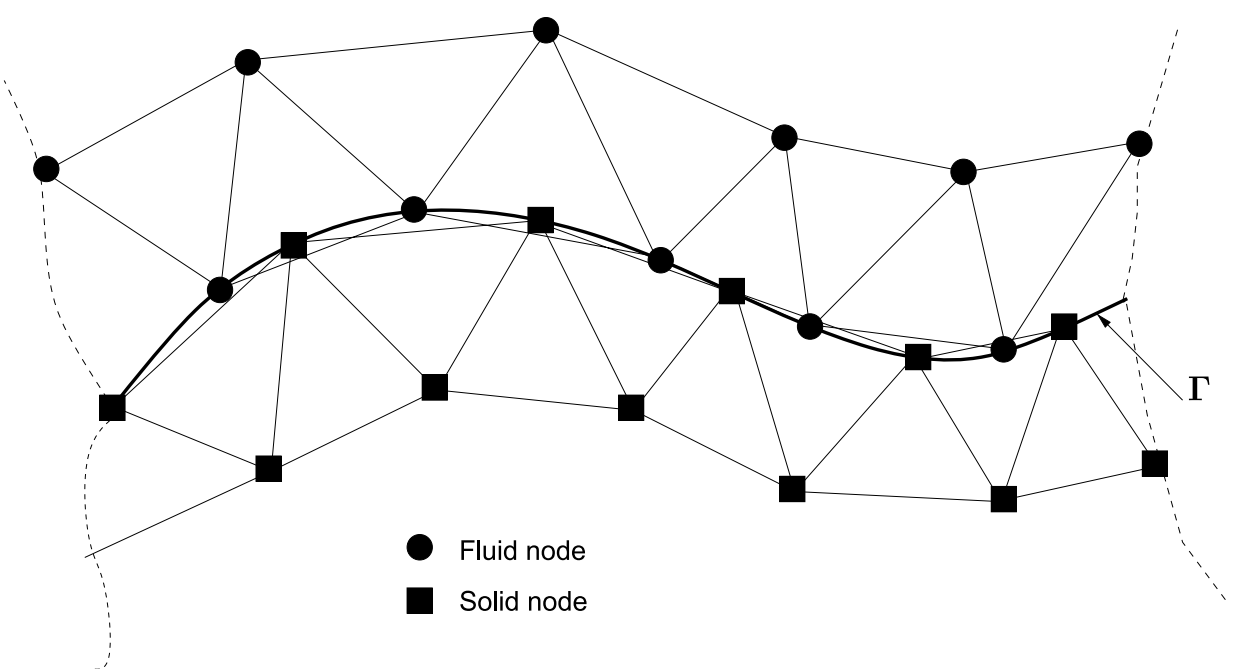

Fig. 1. Schematic of sample region showing non-matching discretization of the same physical interface $\boldsymbol{\Gamma}$. 
Therefore, our emphasis in this paper is to study the accuracy of conservative load transfer along curved interfaces in coupled-field problems with the aid of an explicit 2D loosely coupled fluid-solid interaction (FSI) framework using a partitioned approach. The framework combines a compressible fluid solver, an explicit elasto-dynamic solid solver, and the aforementioned conservative load transfer schemes. The scope of the present work is to remove the necessity of matching meshes and to redesign a more general projection scheme for non-matching fluid and solid nodes along curved interfaces. We consider only 2D problems with smooth curved interfaces for simplicity. However, this study may extend to 3D and complex geometries [14].

The outline of this paper is as follows: Section 2 summarizes the interface coupling conditions and the three load transfer schemes studied in this work. In Section 3, we propose a concept of reference interface for conservation of energy and justify that satisfying the continuity conditions element-wise is sufficient for the stability of transient FSI simulations. The remainder of the paper presents a detailed comparative study of the spatial accuracy of the load transfer schemes, performed with the aid of a static circular-arc load transfer problem (Section 4), a transient super-seismic shock-bump interaction problem (Section 5) and a more physically realistic blast wave problem (Section 6). A key outcome of this experimental study is the set of simple analytical error indicators introduced in Section 4.

\section{Load transfer schemes: review}

Before addressing load transfer along curved interfaces, we provide for completeness a short description of the interface conditions and the load transfer schemes.

\subsection{Interface conditions}

We consider a coupled FSI model, which consists of a fluid domain $\Omega_{\mathrm{f}}$, a solid domain $\Omega_{\mathrm{s}}$, and a common interface boundary $\Gamma=\partial \Omega_{\mathrm{f}} \cap \partial \Omega_{\mathrm{s}}$. At each FSI cycle, two interface boundary conditions corresponding to the continuity of tractions and velocities must be satisfied along $\Gamma$. Let $\boldsymbol{t}_{\mathrm{f}}$ and $\boldsymbol{u}_{\mathrm{f}}$ denote the fluid traction vector and displacement fields along the fluid interface $\Gamma_{\mathrm{f}}$, while $\boldsymbol{t}_{\mathrm{s}}$ and $\boldsymbol{u}_{\mathrm{s}}$ denote the solid traction vector and displacement fields along the solid interface $\Gamma_{\mathrm{s}}$, respectively. The equilibrium of tractions and compatibility of velocity field can be expressed as

$$
\boldsymbol{t}_{\mathrm{s}}=\boldsymbol{t}_{\mathrm{f}}, \quad \frac{\partial \boldsymbol{u}_{\mathrm{s}}}{\partial t}=\frac{\partial \boldsymbol{u}_{\mathrm{f}}}{\partial t} \quad \text { on } \Gamma,
$$

where $\boldsymbol{t}_{\mathrm{f}}=p_{\mathrm{f}} \boldsymbol{n}_{\mathrm{f}}-\boldsymbol{\sigma}_{\mathrm{f}} \cdot \boldsymbol{n}_{\mathrm{f}}$ and $\boldsymbol{t}_{\mathrm{s}}=\boldsymbol{\sigma}_{\mathrm{s}} \cdot \boldsymbol{n}_{\mathrm{s}}$. Here, $p_{\mathrm{f}}$ is the fluid pressure along the interface; $\boldsymbol{\sigma}_{\mathrm{f}}$ and $\boldsymbol{\sigma}_{\mathrm{s}}$ are the fluid viscous tensor and solid stress tensor, respectively; $\boldsymbol{n}_{\mathrm{f}}$ and $\boldsymbol{n}_{\mathrm{s}}$ are the unit outward normals along the fluid interface $\Gamma_{\mathrm{f}}$ and solid interface $\Gamma_{\mathrm{s}}$, respectively.

To consider the discrete problem, let $N_{\mathrm{f}}^{i}$ and $N_{\mathrm{s}}^{j}$ denote the standard finite element shape functions associated with node $i$ of the fluid and node $j$ of the solid interface mesh, respectively, and $\tilde{\boldsymbol{t}}_{\mathrm{f}}^{i} \in L^{2}\left(\Omega_{\mathrm{f}}\right)$ and $\tilde{\boldsymbol{t}}_{\mathrm{s}}^{j} \in L^{2}\left(\Omega_{\mathrm{s}}\right)$ the approximate tractions at the corresponding nodes of the discrete fluid interface $\Gamma_{\mathrm{f}}^{h}$ and solid interface $\Gamma_{\mathrm{f}}^{h}$. Let $m_{\mathrm{f}}$ and $m_{\mathrm{s}}$ be the number of fluid and solid nodes on the fluid and solid interface meshes, respectively. The continuum traction fields $\boldsymbol{t}_{\mathrm{f}}$ and $\boldsymbol{t}_{\mathrm{s}}$ over $\Gamma_{\mathrm{f}}$ and $\Gamma_{\mathrm{s}}$ are interpolated as

$$
\boldsymbol{t}_{\mathrm{f}}(\boldsymbol{x}) \approx \sum_{i=1}^{m_{\mathrm{f}}} N_{\mathrm{f}}^{i}(\boldsymbol{x}) \tilde{\boldsymbol{t}}_{\mathrm{f}}^{i}, \quad \boldsymbol{t}_{\mathrm{s}}(\boldsymbol{x}) \approx \sum_{j=1}^{m_{\mathrm{s}}} N_{\mathrm{s}}^{j}(\boldsymbol{x}) \tilde{\boldsymbol{t}}_{\mathrm{s}}^{j} .
$$

The transfer of distributed loads consists therefore in solving for $\tilde{\boldsymbol{t}}_{\mathrm{s}}^{j}$, given $\tilde{\boldsymbol{t}}_{\mathrm{f}}^{i}, N_{\mathrm{f}}^{i}$, and $N_{\mathrm{s}}^{j}$. To obtain good approximations for $\tilde{\boldsymbol{t}}_{\mathrm{s}}$, we minimize the $L_{2}$ norm of the residual $\boldsymbol{t}_{\mathrm{s}}-\boldsymbol{t}_{\mathrm{f}}$, i.e.,

$$
\left\|\boldsymbol{t}_{\mathrm{s}}-\boldsymbol{t}_{\mathrm{f}}\right\|_{2}=\sqrt{\int_{\Gamma}\left(\boldsymbol{t}_{\mathrm{s}}-\boldsymbol{t}_{\mathrm{f}}\right)^{T}\left(\boldsymbol{t}_{\mathrm{s}}-\boldsymbol{t}_{\mathrm{f}}\right) \mathrm{d} \Gamma .}
$$

This minimization problem can be solved with the aid of the Galerkin weighted residual method [16] by multiplying both sides by a set of weighting functions $\left(W^{i}=N_{\mathrm{s}}^{i}\right)$ and integrating over the interface boundary $\Gamma$, i.e., 


$$
\int_{\Gamma} N_{\mathrm{s}}^{i} \boldsymbol{t}_{\mathrm{s}} \mathrm{d} \Gamma=\int_{\Gamma} N_{\mathrm{s}}^{i} \boldsymbol{t}_{\mathrm{f}} \mathrm{d} \Gamma
$$

Let us insert the finite element approximations for the tractions in (4):

$$
\int_{\Gamma} N_{\mathrm{s}}^{i} N_{\mathrm{s}}^{j} \tilde{\boldsymbol{t}}_{\mathrm{s}}^{j} \mathrm{~d} \Gamma=\int_{\Gamma} N_{\mathrm{s}}^{i} N_{\mathrm{f}}^{j} \tilde{\boldsymbol{t}}_{\mathrm{f}}^{j} \mathrm{~d} \Gamma
$$

and solve for the solid tractions $\tilde{\boldsymbol{t}}_{\mathrm{s}}$ as

$$
\tilde{\boldsymbol{t}}_{\mathrm{s}}^{i}=\left[\boldsymbol{M}_{\mathrm{s}}^{i j}\right]^{-1}\left\{\mathscr{R}_{\mathrm{s}}^{j}\right\},
$$

where $\left[\boldsymbol{M}_{\mathrm{S}}\right]$ is the consistent mass matrix for the solid interface elements and is defined by

$$
\left[\boldsymbol{M}_{\mathrm{s}}^{i j}\right]=\int_{\Gamma} N_{\mathrm{s}}^{i} N_{\mathrm{s}}^{j} \mathrm{~d} \Gamma
$$

and $\left\{\mathscr{R}_{\mathrm{s}}\right\}$ is the concentrated force vector,

$$
\left\{\mathscr{R}_{\mathrm{s}}^{j}\right\}=\sum_{i=1}^{m s} \tilde{\boldsymbol{t}}_{\mathrm{f}}^{i} \int_{\Gamma} N_{\mathrm{f}}^{i} N_{\mathrm{s}}^{j} \mathrm{~d} \Gamma .
$$

Note that, in typical FSI applications, it is sufficient to evaluate (8) for the nodal load vector [10] without solving (6) for the traction vector $\tilde{\boldsymbol{t}}_{\mathrm{s}}$. We concentrate now on the schemes that can be used to evaluate (8), which involves the shape functions on the solid side and the approximated traction field of fluid side. Similar treatment of the interface displacement condition can be derived using the velocity continuity condition. For the evaluation of the energy, the definition of the virtual work can be conveniently used [10], i.e., the conservation of energy can be achieved by an appropriate combination of load and displacement transfer.

There are two essential requirements for transferring methods along the non-matching discrete interface. The first is weak continuity of the traction and displacement fields (i.e., patch test). A method passes the patch test if an arbitrary element patch on one mesh can represent a state of constant field for applied constant field on another mesh. The second requirement pertains to satisfying the conservation of energy along the discrete interface. These two conditions play a key role on the stability of transient FSI simulations. The following subsection describes the load transfer schemes for evaluating the nodal load vector.

\subsection{Load transfer schemes}

As mentioned earlier, conservative load transfer schemes used for generic non-matching interfaces can be categorized in three distinct classes: point-to-point, point-to-element and common-refinement schemes. In this subsection, we present a brief summary of the point-to-element and the common-refinement schemes.

\subsubsection{Point-to-element projection schemes}

Two point-to-element projection schemes are commonly used in FSI, the node projection (N-P) and the quadrature projection (Q-P), as illustrated in Fig. 2. First proposed in [10], the N-P scheme projects the fluid nodes onto the solid surface element to extract the load vector on the solid interface nodes. In particular, the extracted load vector on the solid surface node is then

$$
\mathscr{R}_{\mathrm{s}}^{j}=\sum_{i=1}^{m_{\mathrm{f}}} N_{\mathrm{s}}^{j}\left(\boldsymbol{x}^{i}\right) \mathscr{R}_{\mathrm{f}}^{i},
$$

where $\boldsymbol{x}^{i}$ denotes the location of node $i$ of the fluid interface mesh.

Similar to the N-P approach, the Q-P scheme proposed in [11] projects the fluid quadrature points onto the solid surface element to extract $\boldsymbol{t}_{\mathrm{s}}$ and $\mathscr{R}_{\mathrm{s}}$ on the solid boundary (Fig. 2). Specifically, $\mathscr{R}_{\mathrm{s}}$ is evaluated by

$$
\mathscr{R}_{\mathrm{s}}^{j}=\sum_{i=1}^{e_{\mathrm{f}}} \int_{\sigma_{\mathrm{f}}^{i}} N_{\mathrm{s}}^{j} \tilde{\boldsymbol{t}}_{\mathrm{f}} \mathrm{d} \Gamma,
$$




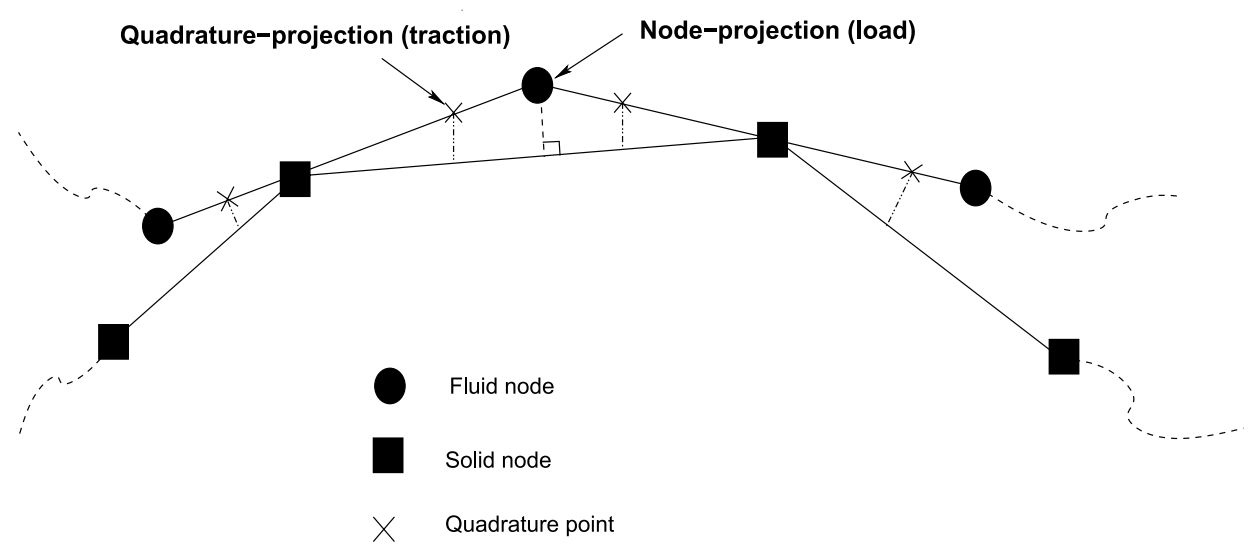

Fig. 2. Schematic of point-to-element projection schemes for load transfer.

where $e_{\mathrm{f}}$ denotes the number of elements on the fluid interface mesh and $\sigma_{\mathrm{f}}^{i}$ its $i$ th element. It can be considered as a rough discretization of the Galerkin weighted residual method, where the integration is performed piecewise over the fluid interface elements with numerical quadrature. To avoid the situation in which a solid target element would receive no load, this scheme may rely on an adaptive quadrature rule by adaptively increasing the number of quadrature points. Further details about this load transfer scheme can be found in [11]. Both point-to-element transfer schemes are conservative but do not pass the traction continuity test due to the inaccurate transfer of loads or tractions from the source fluid mesh to the target solid mesh, as illustrated in [13] by a constant loading on one mesh and transferring it to another mesh. Hence, such schemes generally lead to local errors for non-matching meshes while satisfying the conservation of energy across the distinct interface meshes.

\subsubsection{Common-refinement based scheme}

This scheme defines a common refinement on a reference surface $\Gamma_{\mathrm{r}}$ of the fluid-solid interface. In the finite element form, the spatial configuration of the fluid and solid interface meshes can be parameterized as

$$
\boldsymbol{x}_{\mathrm{f}} \approx \sum_{i=1}^{m_{\mathrm{f}}} N_{\mathrm{f}}^{i}(\boldsymbol{x}) \tilde{\boldsymbol{x}}_{\mathrm{f}}^{i} \text { on } \Gamma_{\mathrm{f}}^{h}, \quad \boldsymbol{x}_{\mathrm{s}} \approx \sum_{j=1}^{m_{\mathrm{s}}} N_{\mathrm{s}}^{j}(\boldsymbol{x}) \tilde{\boldsymbol{x}}_{\mathrm{s}}^{j} \text { on } \Gamma_{\mathrm{s}}^{h} .
$$

In the $2 \mathrm{D}$ common-refinement transfer formulations [13], the reference surface $\Gamma_{\mathrm{r}}$ can be computed by averaging the input meshes on $\Gamma_{\mathrm{f}}^{h}$ and $\Gamma_{\mathrm{s}}^{h}$.

In general, the topology of the common refinement is defined by the intersection of the elements of input meshes, or the "subelements". There can be various situations of intersecting of elements $\sigma_{\mathrm{f}}$ and $\sigma_{\mathrm{s}}$ over $\Gamma_{\mathrm{f}}^{h}$ and $\Gamma_{\mathrm{s}}^{h}$, respectively. For example, in order to determine whether the two elements $\sigma_{\mathrm{f}}$ and $\sigma_{\mathrm{s}}$ intersect, we loop over all the nodes of $\sigma_{\mathrm{f}}$. The two elements intersect if we can find at least one node of $\sigma_{\mathrm{f}}$ that lies inside $\sigma_{\mathrm{s}}$. This can be easily achieved with the aid of the mapping of global to local coordinates available in any finite element code. These subelements are illustrated in Fig. 3(a) for 2D and Fig. 3(b) for 3D. Note that, the intersection of two arbitrary triangles or hybrid surface elements can be quite complex than the situation shown in Fig. 3(b). The intersection of such 3D surface elements is described in [17].

In the common-refinement scheme, the load vector $\mathscr{R}_{\mathrm{s}}^{j}$ over the common-refinement nodes is computed as

$$
\mathscr{R}_{\mathrm{s}}^{j}=\sum_{i=1}^{e_{\mathrm{c}}} \int_{\sigma_{\mathrm{c}}^{i}} N_{\mathrm{s}}^{j} \tilde{\boldsymbol{t}}_{\mathrm{f}} \mathrm{d} \Gamma,
$$

where $e_{\mathrm{c}}$ denotes the number of subelements on the common refinement, and $\sigma_{\mathrm{c}}^{i}$ denotes its $i$ th subelement. The integration point locations and their weight functions can be determined based on Gaussian or Newton-Cotes integration. 
(a)

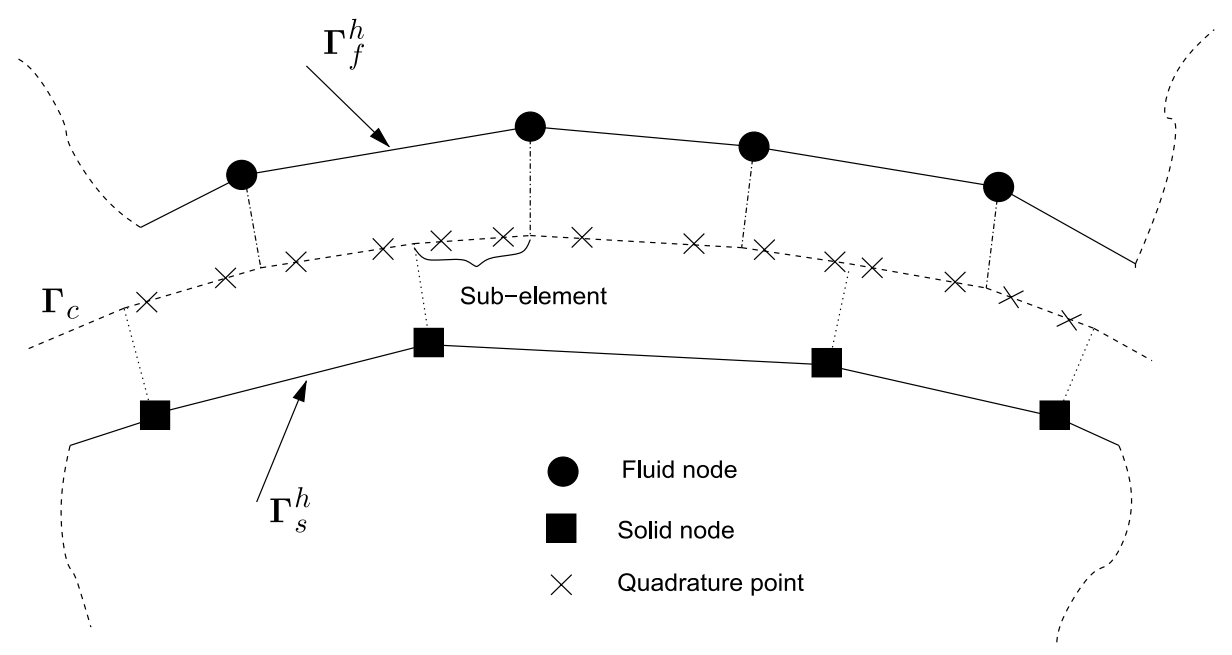

(b)

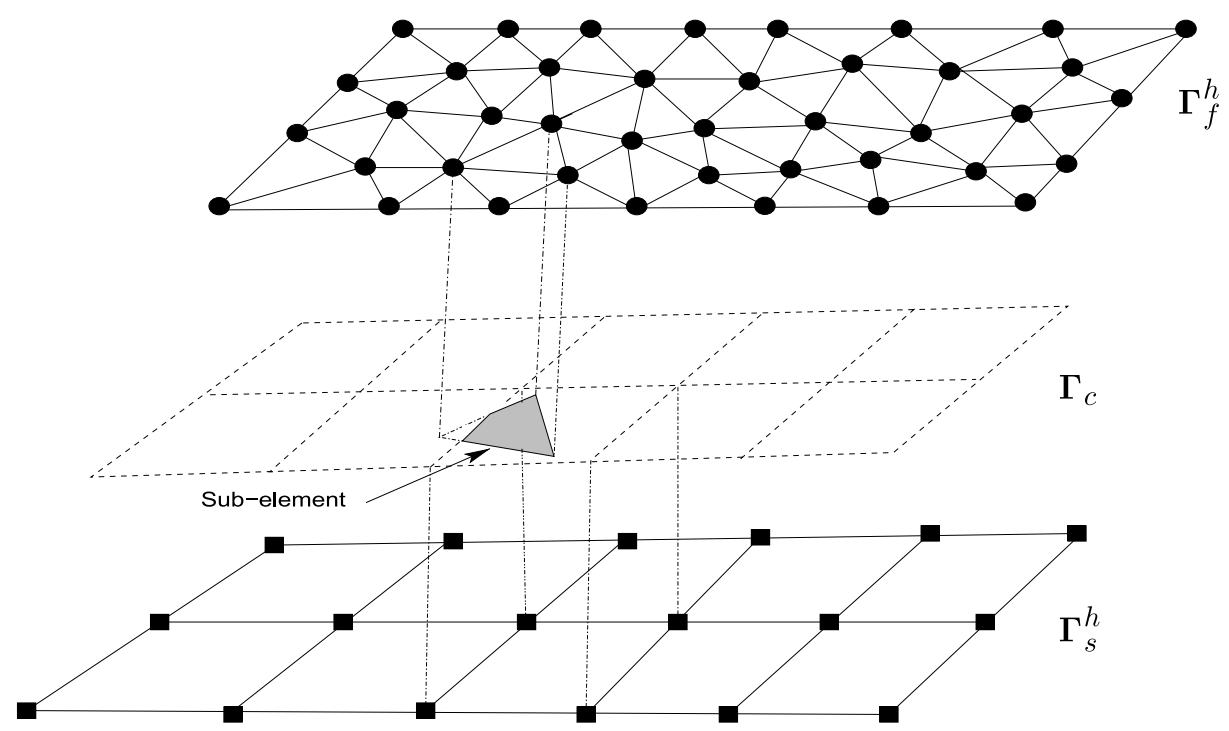

Fig. 3. Schematic of common-refinement based projection scheme for load transfer in (a) 2D and (b) 3D; where shaded area denotes one surface subelement.

\section{Non-matching curved interface}

Let us now turn our attention to the central topic of the present work, i.e., the effect of curvature on the accuracy of load transfer. For simplicity, we restrict our attention to smooth curves in Euclidean space $\mathbb{R}^{2}$. The curvature at a point of a smooth curve, denoted by $\kappa$, is the inverse of the radius of the osculating circle, i.e., the circle that best approximates the curve locally. The curvature captures the rate of variation of normal directions along the curve and is a local property of its shape invariant under Euclidean motions.

As discussed previously, a primary difficulty of load transfer along curved non-matching discretized interfaces is that the fluid and solid meshes typically define two distinct discretizations of the interface. These discretizations will, in general, produce discrepancies in normal directions and areas as shown in Fig. 4. These discrepancies may affect the accuracy of load transfer. The non-matching discrete curved interfaces may also 


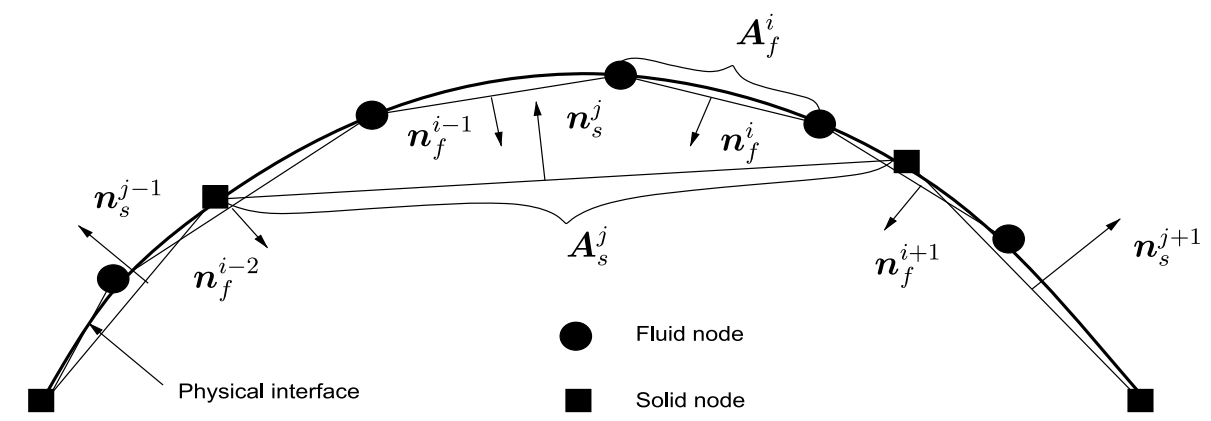

Fig. 4. A portion of smooth curved interface which has inconsistencies in normals and areas in two different discretization.

arise due to geometric interpolation errors, and the discrepancies may increase due to accumulation of errors during coupled simulations. These issues can be even more profound in 3D.

\subsection{Normals and reference interface}

From the interface conditions, it is important that the domains neither detach nor overlap during deformation. Furthermore, in the physical setting, the traction field must be continuous across the fluid-solid interface. The equilibrium condition along the continuous interface for an inviscid fluid simply reads:

$$
\sigma\left(\boldsymbol{u}_{\mathrm{s}}\right) \boldsymbol{n}_{\mathrm{s}}=p \boldsymbol{n}_{\mathrm{f}} \quad \text { on } \Gamma
$$

where $\sigma\left(\boldsymbol{u}_{\mathrm{s}}\right)$ is the stress tensor on the solid side and is a function of the displacement field $\boldsymbol{u}_{\mathrm{s}}, p$ is the fluid pressure, and $\boldsymbol{n}_{\mathrm{f}}$ and $\boldsymbol{n}_{\mathrm{s}}$ are the local normals to the fluid-solid interface. The normals are related by

$$
\boldsymbol{n}_{\mathrm{f}}=-\boldsymbol{n}_{\mathrm{s}} \text { on } \Gamma \text {. }
$$

The normal to a continuous interface is uniquely defined in a geometric sense in the case of smooth surfaces. However, the calculation of the normal to a discretized interface is more challenging as the information about the exact curved interface $\Gamma$ is typically lost and only the computational meshes are usually available.

In the point-to-element projection scheme, the normals are computed on one side of the interface and are then projected onto the target interface elements (Fig. 5(a)). Typically, the solid interface $\Gamma_{\mathrm{s}}^{h}$ is completely ignored in this process. In the point-to-element projection scheme, the direction of the node normal can be computed by averaging surrounding normal vectors to the edges. As shown in Fig. 5(a), the mapped normal vector field over solid elements is discontinuous at the middle fluid node, and the projected segment $\left[p_{1}, p_{2}\right]$ is considered twice while projecting fluid edge normals directly. Due to this lack of continuity at $p_{\text {avg }}$, this type of normal computations may lead to the onset of spurious oscillations in transferring the discretized normals. Therefore, such schemes may fail to satisfy equilibrium even for a uniform fluid pressure and may cause

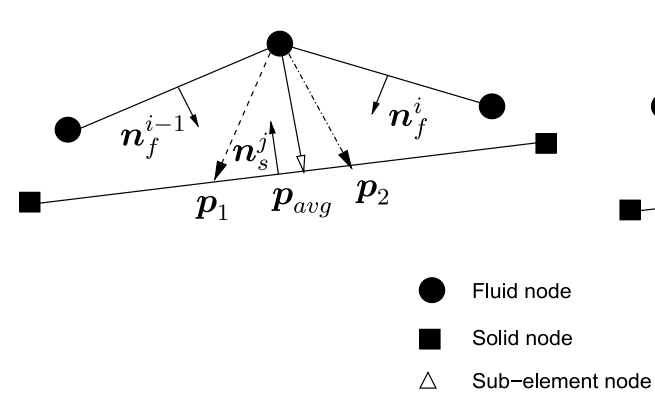

(a)

(b)

Fig. 5. (a) Discontinuity of normal projection in point-to-element transfer scheme, (b) regularity of normal $\boldsymbol{n}_{\mathrm{c}}$ projections via commonrefinement subelements. 
non-physical acceleration of the interface during the simulations. We will analyze errors in the normal transfer with the aid of a patch test in Section 4.

In the common-refinement based scheme, the subelements are generally defined on two discrete curves, which may have different areas and normal directions. To avoid ambiguity in the normals, we define a consistent common normal direction $\boldsymbol{n}_{\mathrm{c}}$ as a linear combination of $\boldsymbol{n}_{\mathrm{f}}$ and $\boldsymbol{n}_{\mathrm{s}}$. A typical normal projection of the subelements is shown in Fig. 5(b). There can be several variants of common-refinement based methods, which utilizes different combinations of the normals and the subelement areas for the load transfer. If the fluid and the solid nodes do not lie on the same smooth surface due to modeling or simulation errors, we can construct an intermediate reference surface using both sides of normals and areas. Herein, we present two extreme variants of common-refinement based discretization for the smooth surfaces with accurate geometric modeling: (a) source-based and (b) target-based discretizations.

The first variant is a source-based discretization, which has the subelements defined over the source interface mesh. The basic steps to be performed in the source-based discretization are summarized as follows:

1. Loop over subelements defined over fluid interface $\Gamma_{\mathrm{f}}^{h}$;

2. Loop over quadrature points of the subelements;

(a) Interpolate fluid traction and normals at the subelement quadrature points;

(b) Compute subelement area over fluid interface;

(c) Associate quadrature points onto corresponding solid element;

(d) Integrate traction vectors using areas of source-based subelements;

3. Compute solid load vector integral using (8);

4. End loop over the subelements.

This type of the common-refinement algorithm satisfies conservation of loads over non-matching interface meshes because it integrates (6) and (8) on different meshes which may have discrepancies in areas and normals. However, this projection scheme is not independent of mesh sizes across the curved interfaces similar to the point-to-element projection schemes. Therefore, it does not preserve exact continuity and compatibility conditions along the non-matching curved interface.

The second variant, referred to as target-based discretization, has the subelements defined over the target interface mesh $\Gamma_{\mathrm{s}}^{h}$. The key difference in this type of discretization compared to source-based discretization is in performing the integration of traction vectors using the areas of target-based subelements. This type of the common-refinement schemes minimizes the error exactly over the target solid interface in a weak sense as the integral

$$
\int_{\Gamma_{\mathrm{s}}^{h}} \phi\left(\sigma\left(\boldsymbol{u}_{\mathrm{s}}\right) \boldsymbol{n}_{\mathrm{s}}-p \boldsymbol{n}_{\mathrm{f}}\right) \quad \mathrm{d} \Gamma=0
$$

for the standard basis functions $\phi$ defined on the target solid interface mesh $\Gamma_{\mathrm{s}}^{h}$. Due to differing areas of fluid and solid elements, this scheme may not yield the same integral of traction field over two distinct fluid and solid interface meshes.

The conservation of loads can be satisfied by an infinite number of possible nodal load vectors over the distinct interfaces. To be physically consistent and meaningful, the transformation of load must also be $a c c u$ rate, i.e., to have a small error in a specific norm, and the conservation of energy can be satisfied on a reference interface [12]. Traditional point-to-element projection schemes try to enforce conservation of energy over two distinct surface meshes, which may not yield physically equivalent definitions of the conservation of loads. Furthermore, such schemes may lead to unbounded local errors along the interface [13].

A simple and reasonable choice of the reference surface is a convex combination of the input interface meshes, i.e., $\Gamma_{\mathrm{r}}=\alpha \Gamma_{\mathrm{f}}^{h}+\beta \Gamma_{\mathrm{s}}^{h}$, where $\alpha$ and $\beta$ are two non-negative constants with $\alpha+\beta=1$ [14]. Each mesh point on this reference surface is associated with some point on the underlying source- and target-based discretizations. The fluid traction field and computed solid traction field are projected onto this reference interface, upon which the load conservation is defined in the $L^{1}$-norm as 


$$
\left\|\sigma\left(\boldsymbol{u}_{\mathrm{s}}\right) \boldsymbol{n}_{\mathrm{s}}\right\|_{L^{1}\left(\Gamma_{\mathrm{r}}\right)}=\left\|p \boldsymbol{n}_{\mathrm{f}}\right\|_{L^{1}\left(\Gamma_{\mathrm{r}}\right)},
$$

where $\|\cdot\|_{L^{1}\left(\Gamma_{\mathrm{r}}\right)}=\int_{\Gamma_{\mathrm{\Gamma}}}[\cdot] \mathrm{d} \Gamma$. Therefore, the computation of load conservation over the reference interface is satisfied by the integral

$$
\int_{\Gamma_{\mathrm{r}}} \psi\left[\left\|\sigma\left(\boldsymbol{u}_{\mathrm{s}}\right) \boldsymbol{n}_{\mathrm{s}}\right\|_{L^{1}\left(\Gamma_{\mathrm{r}}\right)}-\left\|p \boldsymbol{n}_{\mathrm{f}}\right\|_{L^{1}\left(\Gamma_{\mathrm{r}}\right)}\right] \mathrm{d} \Gamma=0
$$

for the standard basis functions $\psi$ defined on the reference interface mesh $\Gamma_{\mathrm{r}}$. For complex non-convex geometries, it may become difficult to select a reasonable mapping for all points on the reference surface to the common-refinement discretizations.

\subsection{Stability and conservation}

We now address the issues of the energy conservation error and stability of the loosely-coupled iterative algorithm defined on the reference interface. In particular, we show that satisfying the continuity conditions element-wise is sufficient for the stability of the coupled simulations for a simple explicit staggered time stepping procedure [18]. Since there is no established nonlinear stability condition for analyzing the solution of the nonlinear Euler and Navier-Stokes equations, we consider a linear fluid-solid interaction model based on the linearized Euler (acoustic) and linear elastodynamic equations with well-posed initial and boundary conditions.

\subsubsection{Coupled system}

We first present the formulation of the linear acoustic equation in terms of the pressure function $p$. The boundary of $\Omega_{\mathrm{f}}$ is the union of disjoint subsets $\Gamma_{\mathrm{f}}^{\mathrm{n}}$ and $\Gamma_{\mathrm{f}}^{\mathrm{e}}$, on which natural and essential data are given, respectively. Denoting the speed of sound by $c$, the strong formulation for linear acoustics reads as follows: Given $g_{\mathrm{f}}, p_{0}, \frac{\partial_{p_{0}}}{\partial_{t}}: \Omega_{\mathrm{f}} \rightarrow \mathbb{R}$, find $p: \bar{\Omega}_{\mathrm{f}} \times[0, T] \rightarrow \mathbb{R}$ such that

$$
\frac{1}{c^{2}} \frac{\partial^{2} p}{\partial t^{2}}-\nabla^{2} p=g_{\mathrm{f}} \quad \text { in } \Omega_{\mathrm{f}} \times(0, T)
$$

with boundary conditions

$$
p=p_{e} \text { on } \Gamma_{\mathrm{f}}^{\mathrm{e}} \times(0, T), \quad \frac{\partial p}{\partial n_{\mathrm{f}}}=p_{n} \text { on } \Gamma_{\mathrm{f}}^{\mathrm{n}} \times(0, T),
$$

and initial conditions

$$
p(\boldsymbol{x}, 0)=p_{0}, \frac{\partial p(\boldsymbol{x}, 0)}{\partial t}=\frac{\partial p_{0}}{\partial t}, \quad \boldsymbol{x} \in \Omega_{\mathrm{f}} .
$$

Similarly, we investigate the deformation of an elastic body $\Omega_{\mathrm{s}}$ with density $\rho_{\mathrm{s}}$ under given time-dependent volume and surface forces denoted by $\boldsymbol{g}_{\mathrm{s}}$ and $\boldsymbol{g}_{n}$. The strong formulation for elastodynamic problems then reads as follows: Given $\boldsymbol{g}_{\mathrm{s}}, \boldsymbol{u}_{0}, \frac{\partial \boldsymbol{u}_{0}}{\partial t}: \Omega_{\mathrm{s}} \rightarrow \mathbb{R}$, find $\boldsymbol{u}_{\mathrm{s}}: \bar{\Omega}_{\mathrm{s}} \times[0, T] \rightarrow \mathbb{R}$ such that

$$
\rho_{\mathrm{s}} \frac{\partial^{2} \boldsymbol{u}_{\mathrm{s}}}{\partial t^{2}}-\operatorname{div}\left(\sigma\left(\boldsymbol{u}_{\mathrm{s}}\right)\right)=\boldsymbol{g}_{\mathrm{s}} \quad \text { in } \Omega_{\mathrm{s}} \times(0, T),
$$

with boundary conditions

$$
\boldsymbol{u}_{\mathrm{s}}=\boldsymbol{u}_{\mathrm{s}}^{\mathrm{e}} \text { on } \Gamma_{\mathrm{s}}^{\mathrm{e}} \times(0, T), \quad\left[\sigma\left(\boldsymbol{u}_{\mathrm{s}}\right)\right] \cdot \boldsymbol{n}_{\mathrm{s}}=\boldsymbol{g}_{n} \text { on } \Gamma_{\mathrm{s}}^{\mathrm{n}} \times(0, T),
$$

and initial conditions

$$
\boldsymbol{u}(\boldsymbol{x}, 0)=\boldsymbol{u}_{0}, \frac{\partial \boldsymbol{u}(\boldsymbol{x}, 0)}{\partial t}=\frac{\partial \boldsymbol{u}_{0}}{\partial t}, \quad \boldsymbol{x} \in \Omega_{\mathrm{s}}
$$

Since an acoustic medium can be considered as an elastic medium with zero shear modulus [19], the motion of each part of the composite medium can be described by a system of elastic wave equations: 


$$
\begin{aligned}
& \rho_{i} \frac{\partial^{2}\left(\boldsymbol{u}_{i}\right)}{\partial t^{2}}=\operatorname{div} \sigma\left(\boldsymbol{u}_{i}\right)+\boldsymbol{g}_{i} \text { in } \Omega_{i}, \\
& \sigma\left(\boldsymbol{u}_{i}\right)=\lambda_{i} \operatorname{div}\left(\boldsymbol{u}_{i}\right) I+2 \mu_{i} \epsilon\left(\boldsymbol{u}_{i}\right), \\
& \epsilon\left(\boldsymbol{u}_{i}\right)=\frac{1}{2}\left[\nabla \boldsymbol{u}_{i}+\left(\nabla \boldsymbol{u}_{i}\right)^{\mathrm{T}}\right],
\end{aligned}
$$

where $i=\mathrm{f}$, s. In $\Omega_{i}, \boldsymbol{u}_{i}$ denotes the displacement vector, $\lambda_{i}$, and $\mu_{i}$ are the Lamé constants (with $\mu_{\mathrm{f}}=0$ ), $\rho_{i}$, denotes the density, $\boldsymbol{g}_{i}$ denote source terms, and $I$ stands for the identity tensor. $\sigma$ and $\epsilon$ are the stress and strain tensors, respectively. In the fluid domain, $\sigma \equiv p I$. This coupled model is physically stable, i.e., has a solution that does not grow indefinitely in time. The objective herein is to present a mathematical framework for the stability analysis of the spatial interfacing methods across the non-matching discretization. We assume that there is no numerical energy dissipation in the discrete time and space and that each system is stable under standard CFL conditions.

\subsubsection{Transformation of interface conditions}

To derive the stability condition of the coupled model, we must first transform the interface conditions using the above pressure-displacement formulation (20)-(22). From (20), the velocity continuity can be expressed as

$$
\rho_{\mathrm{f}} \frac{\partial^{2}\left(\boldsymbol{u}_{\mathrm{s}} \cdot \boldsymbol{n}_{\mathrm{s}}\right)}{\partial t^{2}}=\rho_{\mathrm{f}} \frac{\partial^{2}\left(\boldsymbol{u}_{\mathrm{f}} \cdot \boldsymbol{n}_{\mathrm{f}}\right)}{\partial t^{2}}=\operatorname{div}\left(\sigma\left(\boldsymbol{u}_{\mathrm{f}}\right)\right) \cdot \boldsymbol{n}_{\mathrm{f}}=\frac{\partial p}{\partial n_{\mathrm{f}}} \quad \text { on } \Gamma
$$

which implies that

$$
\frac{\partial p}{\partial n_{\mathrm{f}}}=\rho_{\mathrm{f}} \frac{\partial^{2} \boldsymbol{u}_{\mathrm{s}}}{\partial t^{2}} \cdot \boldsymbol{n}_{\mathrm{s}} \quad \text { on } \Gamma
$$

Here we have used the fact the source $\boldsymbol{g}_{\mathrm{f}}$ vanishes on the interface $\Gamma$, and $\boldsymbol{n}_{\mathrm{s}}$ and $\boldsymbol{n}_{\mathrm{f}}$ along $\Gamma$ are time-invariant for the infinitesimal deformation. From the conventional interface condition (23), we derive a transformed interface condition:

$$
\rho_{\mathrm{f}} \frac{\partial^{2} \boldsymbol{u}_{\mathrm{s}}}{\partial t^{2}}+\omega \sigma\left(\frac{\partial \boldsymbol{u}_{\mathrm{s}}}{\partial t}\right) \boldsymbol{n}_{\mathrm{s}}=\frac{\partial p}{\partial n_{\mathrm{f}}} \boldsymbol{n}_{\mathrm{s}}-\omega \frac{\partial p}{\partial t} \boldsymbol{n}_{\mathrm{s}} \quad \text { on } \Gamma
$$

where (25) is obtained by differentiating (13) with respect to $t$, i.e.,

$$
\sigma\left(\frac{\partial \boldsymbol{u}_{\mathrm{s}}}{\partial t}\right) \boldsymbol{n}_{\mathrm{s}}=\frac{\partial p}{\partial t} \boldsymbol{n}_{\mathrm{f}}
$$

multiplying with a positive dimensional constant $\omega$ (to make the combination dimensionally consistent), using (14), and then adding with (23). Similarly, we obtain a transformed representation of (24) by adding the relation $\frac{\partial p}{\partial t}=-\sigma\left(\frac{\partial u_{\mathrm{s}}}{\partial t}\right) \boldsymbol{n}_{\mathrm{s}} \cdot \boldsymbol{n}_{\mathrm{s}}$ multiplied by $\omega$ :

$$
\frac{\partial p}{\partial n_{\mathrm{f}}}+\omega \frac{\partial p}{\partial t}=\rho f \frac{\partial^{2} \boldsymbol{u}_{\mathrm{s}}}{\partial t^{2}} \cdot \boldsymbol{n}_{\mathrm{s}}-\omega \sigma\left(\frac{\partial \boldsymbol{u}_{\mathrm{s}}}{\partial t}\right) \boldsymbol{n}_{\mathrm{s}} \cdot \boldsymbol{n}_{\mathrm{s}} \quad \text { on } \Gamma .
$$

The new forms (25) and (27) of the interface conditions are constructed for the consistency of the residual interface energy, which is introduced as part of the stability analysis presented next.

\subsubsection{Stability analysis}

For simplicity, we analyze the stability of the linearized Euler and linear elasto-dynamic equations at the differential level, and show that the error in the energy transfer decreases monotonically when the continuity conditions (25) and (27) are satisfied element-wise (i.e., patch test). We also show that the energy conservation is automatically satisfied on the aforementioned reference fluid-solid interface, $\Gamma_{\mathrm{r}}$.

Based on the new form (25) and (27) of the interface conditions, we construct the explicit staggered iterative procedure described in Algorithm 1, which serves as the foundation of our stability analysis. 
Algorithm 1. Explicit staggered scheme for the linearized transient FSI problem

1. Start from initial conditions to (18) and (19)

2. Generate $\left\{p^{n}\right\}_{n \geqslant 0}$ and $\left\{\boldsymbol{u}_{\mathrm{s}}^{n}\right\}_{n \geqslant 1}$ iteratively as follows:

(a) Solve acoustic equation (18)

$$
\frac{1}{c^{2}} \frac{\partial^{2} p^{n}}{\partial t^{2}}-\nabla^{2} p^{n}=g_{\mathrm{f}}^{n} \quad \text { in } \Omega_{\mathrm{f}} \times[0, T]
$$

(b) Solve interface condition (25)

$$
\rho_{\mathrm{f}} \frac{\partial^{2} \boldsymbol{u}_{\mathrm{s}}^{n+1}}{\partial t^{2}}+\omega \sigma\left(\frac{\partial \boldsymbol{u}_{\mathrm{s}}^{n+1}}{\partial t}\right) \boldsymbol{n}_{\mathrm{s}}=\frac{\partial p^{n}}{\partial n_{\mathrm{f}}} \boldsymbol{n}_{\mathrm{s}}-\omega \frac{\partial p^{n}}{\partial t} \boldsymbol{n}_{\mathrm{s}} \quad \text { on } \Gamma_{\mathrm{r}} \times[0, T]
$$

(c) Solve elastodynamic equation (19)

$$
\rho_{\mathrm{s}} \frac{\partial 2 \boldsymbol{u}_{\mathrm{s}}^{n+1}}{\partial t^{2}}-\operatorname{div}\left(\sigma\left(\boldsymbol{u}_{\mathrm{s}}^{n+1}\right)\right)=\boldsymbol{g}_{\mathrm{s}}^{n} \quad \text { in } \Omega_{\mathrm{s}} \times[0, T]
$$

(d) Solve interface condition (27)

$$
\frac{\partial p^{n+1}}{\partial n_{\mathrm{f}}}+\omega \frac{\partial p^{n+1}}{\partial t}=\rho_{\mathrm{f}} \frac{\partial^{2} \boldsymbol{u}_{\mathrm{s}}^{n}}{\partial t^{2}} \cdot \boldsymbol{n}_{\mathrm{s}}-\omega \sigma\left(\frac{\partial \boldsymbol{u}_{\mathrm{s}}^{n}}{\partial t}\right) \boldsymbol{n}_{\mathrm{s}} \cdot \boldsymbol{n}_{\mathrm{s}} \quad \text { on } \Gamma_{\mathrm{r}} \times[0, T]
$$

As in [20], let us introduce the error functions $r$ in the fluid and $e$ in the solid at the $n$th iteration as

$$
r^{n}=p-p^{n}, \quad \boldsymbol{e}^{n}=\boldsymbol{u}_{\mathrm{s}}-\boldsymbol{u}_{\mathrm{s}} .
$$

From linearity of the acoustic and elastodynamic equations, the error functions $\left(r^{n}, e^{n}\right)$ satisfy:

$$
\begin{aligned}
& \frac{1}{c^{2}} \frac{\partial^{2} r^{n}}{\partial t^{2}}-\nabla^{2} r^{n}=0 \quad \text { in } \Omega_{\mathrm{f}} \times[0, T], \\
& \rho_{\mathrm{f}} \frac{\partial^{2} \boldsymbol{e}^{n+1}}{\partial t^{2}} \cdot \boldsymbol{n}_{\mathrm{s}}+\omega \sigma\left(\frac{\partial \boldsymbol{e}^{n+1}}{\partial t}\right) \boldsymbol{n}_{\mathrm{s}} \cdot \boldsymbol{n}_{\mathrm{s}}=\frac{\partial r^{n}}{\partial n_{\mathrm{f}}}-\omega \frac{\partial r^{n}}{\partial t} \quad \text { on } \Gamma_{\mathrm{r}} \times[0, T], \\
& \rho_{\mathrm{s}} \frac{\partial^{2} \boldsymbol{e}^{n+1}}{\partial t^{2}}-\operatorname{div}\left(\sigma\left(\boldsymbol{e}^{n+1}\right)\right)=0 \quad \text { in } \Omega_{\mathrm{s}} \times[0, T], \\
& \frac{\partial r^{n+1}}{\partial n_{\mathrm{f}}}+\omega \frac{\partial r^{n+1}}{\partial t}=\rho_{\mathrm{f}} \frac{\partial^{2} \boldsymbol{e}^{n}}{\partial t^{2}} \cdot \boldsymbol{n}_{\mathrm{s}}-\omega \sigma\left(\frac{\partial \boldsymbol{e}^{n}}{\partial t}\right) \boldsymbol{n}_{\mathrm{s}} \cdot \boldsymbol{n}_{\mathrm{s}} \quad \text { on } \Gamma_{\mathrm{r}} \times[0, T] .
\end{aligned}
$$

Now let us consider the energy state of the interface across non-matching meshes. Physically, the interface should neither produce nor damp the energy during the information transfer in finite time $\tau$. Let us also define the "residual energy" $E^{n+1}$ along the interface for $L^{2}$ integrable fluid pressure and solid displacement as the space and time integral [20]:

$$
E^{n+1}=\left\|\rho_{\mathrm{f}} \frac{\partial^{2} e^{n+1}}{\partial t^{2}} \cdot \boldsymbol{n}_{\mathrm{s}}+\omega \sigma\left(\frac{\partial \boldsymbol{e}^{n+1}}{\partial t}\right) \boldsymbol{n}_{\mathrm{s}} \cdot \boldsymbol{n}_{\mathrm{s}}\right\|_{L^{2}\left((0, \tau), L^{2}\left(\Gamma_{\mathrm{r}}\right)\right)}^{2}+\left\|\frac{\partial r^{n+1}}{\partial n_{\mathrm{f}}}+\omega \frac{\partial r^{n+1}}{\partial t}\right\|_{L^{2}\left((0, \tau), L^{2}\left(\Gamma_{\mathrm{r}}\right)\right)}^{2},
$$

where $\|\cdot\|_{L^{2}\left((0, \tau), L^{2}\left(\Gamma_{\mathrm{r}}\right)\right)}^{2}=\int_{0}^{\tau} \int_{\Gamma_{\mathrm{r}}}[\cdot]^{2} \mathrm{~d} \Gamma \mathrm{d} t$.

Due to the $L_{2}$ minimization involved in the common-refinement scheme (see Eq. (15)), we satisfy the interface conditions (30) and (32) element-wise in a weak sense across non-matching meshes. Note, however, that these interface conditions may not be satisfied by the point-to-element treatment when there is a mismatch between the fluid and solid meshes along the interface [13]. We assume that the load and motion transfer integrals are performed with sufficient accuracy so that in the assessment of stability a numerical integration error can be neglected. Hence, locally accurate load and motion transfer by the common-refinement method allows us to use (30) and (32) to express the residual energy as: 


$$
E^{n+1}=\left\|\frac{\partial r^{n}}{\partial n_{\mathrm{f}}}-\omega \frac{\partial r^{n}}{\partial t}\right\|_{L^{2}\left((0, \tau), L^{2}\left(\Gamma_{\mathrm{r}}\right)\right)}^{2}+\left\|\rho_{\mathrm{f}} \frac{\partial^{2} \boldsymbol{e}^{n}}{\partial t^{2}} \cdot \boldsymbol{n}_{\mathrm{s}}-\omega \sigma\left(\frac{\partial \boldsymbol{e}^{n}}{\partial t}\right) \boldsymbol{n}_{\mathrm{s}} \cdot \boldsymbol{n}_{\mathrm{s}}\right\|_{L^{2}\left((0, \tau), L^{2}\left(\Gamma_{\mathrm{r}}\right)\right)}^{2} .
$$

Through some algebraic manipulations, we rewrite $E^{n+1}$ as:

$$
\begin{aligned}
E^{n+1}= & \underbrace{\left\|\rho_{\mathrm{f}} \frac{\partial^{2} \boldsymbol{e}^{n}}{\partial t^{2}} \cdot \boldsymbol{n}_{\mathrm{s}}+\omega \sigma\left(\frac{\partial \boldsymbol{e}^{n}}{\partial t}\right) \boldsymbol{n}_{\mathrm{s}} \cdot \boldsymbol{n}_{\mathrm{s}}\right\|_{L^{2}\left((0, \tau), L^{2}\left(\Gamma_{\mathrm{r}}\right)\right)}^{2}+\left\|\frac{\partial r^{n}}{\partial n_{\mathrm{f}}}+\omega \frac{\partial r^{n}}{\partial t}\right\|_{L^{2}\left((0, \tau), L^{2}\left(\Gamma_{\mathrm{r}}\right)\right)}^{2}}_{R^{n}} \\
& \underbrace{-4 \omega \int_{0}^{\tau} \int_{\Gamma_{\mathrm{r}}}\left[\frac{\partial r^{n}}{\partial n_{\mathrm{f}}} \frac{\partial r^{n}}{\partial t}+\rho_{\mathrm{f}} \frac{\partial^{2} \boldsymbol{e}^{n}}{\partial t^{2}} \cdot \sigma\left(\frac{\partial \boldsymbol{e}^{n}}{\partial t}\right) \boldsymbol{n}_{\mathrm{s}}\right] \mathrm{d} \Gamma \mathrm{d} t}_{E^{n}},
\end{aligned}
$$

so we obtain the following identity:

$$
E^{n+1}(\tau)=E^{n}(\tau)-R^{n}(\tau) \text { for } n \geqslant 1 .
$$

The term $R^{n}$ consists of products of load and motion transfer error terms and represents the integrated error in residual energy at the $n$th iteration along the reference interface.

We now show that $R^{n}$ has a positive lower bound in terms of the error estimates for the fluid and solid equations. We can find a lower bound for $R^{n}$ by testing (29) against $\frac{\partial r^{n}}{\partial t}$ and assuming $r^{n}(0)=\frac{\partial r^{n}}{\partial t}(0)=0$. For all $\tau \in(0, T]$, we have

$$
\int_{0}^{\tau} \int_{\Gamma_{\mathrm{r}}} \frac{\partial r^{n}}{\partial n_{\mathrm{f}}} \frac{\partial r^{n}}{\partial t} \mathrm{~d} \Gamma \mathrm{d} t=\frac{1}{2}\left\|\frac{1}{c} \frac{\partial r^{n}(\tau)}{\partial t}\right\|_{0, \Omega_{\mathrm{f}}}^{2}+\frac{1}{2}\left\|\nabla r^{n}(\tau)\right\|_{0, \Omega_{\mathrm{f}}}^{2}+\int_{0}^{\tau}\left\|\frac{1}{\sqrt{c}} \frac{\partial r^{n}(\tau)}{\partial t}\right\|_{\left(L^{2}(0, \tau), L^{2}\left(\Gamma_{\mathrm{f}}\right)\right)} .
$$

Similarly, differentiating (31) with respect to $t$ and testing it against $\frac{\partial^{2} e^{n}}{\partial t^{2}}$ leads to

$$
\begin{aligned}
& \int_{0}^{\tau} \int_{\Gamma_{\mathrm{r}}}\left[\rho_{\mathrm{f}} \frac{\partial^{2} \boldsymbol{e}^{n}}{\partial t} \cdot \sigma\left(\frac{\partial \boldsymbol{e}^{n}}{\partial t}\right) \boldsymbol{n}_{\mathrm{s}}\right] \mathrm{d} \Gamma \mathrm{d} t \\
& \quad \geqslant \frac{1}{2}\left\|\sqrt{\rho_{\mathrm{s}}} \frac{\partial^{2} \boldsymbol{e}^{n}(\tau)}{\partial t^{2}}\right\|_{0, \Omega_{\mathrm{s}}}^{2}+\left\|\sqrt{\mu_{\mathrm{s}}} \epsilon\left(\frac{\partial \boldsymbol{e}^{n}(\tau)}{\partial t}\right)\right\|_{0, \Omega_{\mathrm{s}}}^{2}+\frac{1}{2}\left\|\sqrt{\lambda_{\mathrm{s}}} \operatorname{div}\left(\frac{\partial \boldsymbol{e}^{n}(\tau)}{\partial t}\right)\right\|_{0, \Omega_{\mathrm{s}}}^{2}+c_{0}\left\|\sqrt{\rho_{\mathrm{s}}} \frac{\partial \boldsymbol{e}^{n}(\tau)}{\partial t}\right\|_{\left(L^{2}(0, \tau), L^{2}\left(\Gamma_{\mathrm{s}}\right)\right)},
\end{aligned}
$$

where $\boldsymbol{e}^{n}(0)=\frac{\partial e^{n}(0)}{\partial t}=0,\left\|\sqrt{\rho_{\mathrm{s}}} \frac{\partial^{2} e^{n}(0)}{\partial t^{2}}\right\|=\left\|\sqrt{\lambda_{\mathrm{s}}} \operatorname{div}\left(\frac{\partial \boldsymbol{e}(0)^{n}}{\partial t}\right)\right\|=0$ and $c_{0}$ is a positive constant. Every term on the right-hand side of (37) and (38) is a non-negative term. Hence, $R^{n}$ has a positive lower bound and

$$
E^{n+1}(\tau) \leqslant E^{n}(\tau) \text {. }
$$

This stability result, which is similar to that proved in [20] for matching meshes, confirms that the commonrefinement scheme that satisfies the weak continuity and compatibility conditions across non-matching meshes preserves the stability condition.

\subsubsection{Conservation analysis}

Let us return to the minimization-based load conservation relation (16) defined on the reference interface $\Gamma_{\mathrm{r}}$. We now aim to show that total energy conservation error associated with (16) decays with time. To that effect, based on the interface conditions (30) and (32), let us consider the work quantity

$$
W^{n+1}=\left|\int_{\Gamma_{\mathrm{r}}}\left[\rho_{\mathrm{f}} \frac{\partial^{2} \boldsymbol{e}^{n+1}}{\partial t^{2}} \cdot \boldsymbol{n}_{\mathrm{s}}+\omega \sigma\left(\frac{\partial \boldsymbol{e}^{n+1}}{\partial t}\right) \boldsymbol{n}_{\mathrm{s}} \cdot \boldsymbol{n}_{\mathrm{s}}\right]\left[\frac{\partial r^{n+1}}{\partial n_{\mathrm{f}}}+\omega \frac{\partial r^{n+1}}{\partial t}\right] \mathrm{d} \Gamma\right| .
$$

which represents the product of load and motion transfer at the $(n+1)$ th iteration. From the CauchySchwarz inequality, we can express $W^{n+1}$ as 


$$
W^{n+1} \leqslant\left[\int_{\Gamma_{\mathrm{r}}}\left|\left(\rho_{\mathrm{f}} \frac{\partial^{2} \boldsymbol{e}^{n+1}}{\partial t^{2}} \cdot \boldsymbol{n}_{\mathrm{s}}+\omega \sigma\left(\frac{\partial \boldsymbol{e}^{n+1}}{\partial t}\right) \boldsymbol{n}_{\mathrm{s}} \cdot \boldsymbol{n}_{\mathrm{s}}\right)\right| \mathrm{d} \Gamma\right]\left[\int_{\Gamma_{\mathrm{r}}}\left|\left(\frac{\partial r^{n+1}}{\partial n_{\mathrm{f}}}+\omega \frac{\partial r^{n+1}}{\partial t}\right)\right| \mathrm{d} \Gamma\right],
$$

where the right-hand side (41), denoted hereafter by $I^{n+1}$, represents an upper bound for the net energy conservation error. Combining (16) with (30) and (32), we get

$$
I^{n+1}=\left[\int_{\Gamma_{\mathrm{r}}}\left|\left(\frac{\partial r^{n}}{\partial n_{\mathrm{f}}}-\omega \frac{\partial r^{n}}{\partial t}\right)\right| \mathrm{d} \Gamma\right] \times\left[\int_{\Gamma_{\mathrm{r}}}\left|\left(\rho_{\mathrm{f}} \frac{\partial^{2} \boldsymbol{e}^{n}}{\partial t^{2}} \cdot \boldsymbol{n}_{\mathrm{s}}-\omega \sigma\left(\frac{\partial \boldsymbol{e}^{n}}{\partial t}\right) \boldsymbol{n}_{\mathrm{s}} \cdot \boldsymbol{n}_{\mathrm{s}}\right)\right| \mathrm{d} \Gamma\right] .
$$

For any positive and bounded perturbation terms $\epsilon$ and $\delta$, we have $\|f-\epsilon\|_{L^{1}\left(\Gamma_{\mathrm{r}}\right)}\|g-\delta\|_{L^{1}\left(\Gamma_{\mathrm{r}}\right)} \leqslant$ $\|f\|_{L^{1}\left(\Gamma_{\mathrm{r}}\right)}\|g\|_{L^{1}\left(\Gamma_{\mathrm{r}}\right)}$, where $f$ and $g$ are $\left|\left(\frac{\partial r^{n}}{\partial n_{\mathrm{f}}}+\omega \frac{\partial r^{n}}{\partial t}\right)\right|$ and $\left|\left(\rho_{\mathrm{f}} \frac{\partial^{2} e^{n}}{\partial t^{2}} \cdot \boldsymbol{n}_{\mathrm{S}}+\omega \sigma\left(\frac{\partial e^{n}}{\partial t}\right) \boldsymbol{n}_{\mathrm{s}} \cdot \boldsymbol{n}_{\mathrm{s}}\right)\right|$, respectively. It is now easy to show using (37) and (38) that

$$
I^{n+1} \leqslant\left[\int_{\Gamma_{\mathrm{r}}}\left|\left(\frac{\partial r^{n}}{\partial n_{\mathrm{f}}}+\omega \frac{\partial r^{n}}{\partial t}\right)\right| \mathrm{d} \Gamma\right] \times\left[\int_{\Gamma_{\mathrm{r}}}\left|\left(\rho_{\mathrm{f}} \frac{\partial^{2} \boldsymbol{e}^{n}}{\partial t^{2}} \cdot \boldsymbol{n}_{\mathrm{s}}+\omega \sigma\left(\frac{\partial \boldsymbol{e}^{n}}{\partial t}\right) \boldsymbol{n}_{\mathrm{s}} \cdot \boldsymbol{n}_{\mathrm{s}}\right)\right| \mathrm{d} \Gamma\right],
$$

from which it immediately follows that

$$
I^{n+1} \leqslant I^{n},
$$

i.e., the resulting integrated error in the energy transfer $I$ at the $(n+l)$ th iteration is found to be always less than that at the $n$th iteration along the reference interface constructed by the common-refinement scheme.

The stability and conservation of energy results are of practical interest to many fluid-solid interaction applications where the local errors along the interface caused by the point-to-element schemes can lead to spurious oscillations and overshoots in the coupled simulations. To assess further the accuracy and stability associated with the point-to-element and common-refinement load transfer schemes, we next turn our attention to three test problems of increasing complexity: the first problem involves a static circular interface with varying mesh resolutions while the other two consist of transient fluid-solid interaction problems with deforming interfaces. As mentioned earlier, our numerical study focuses on 2D FSI problems for simplicity. The comparative assessment of the precision of the load transfer schemes is however expected to generalize to higher dimensions.

\section{Static circular-arc problem}

The first model problem used to assess the effects of grid mismatch and curvature on the accuracy of the conservative load transfer schemes consists of the semicircular interface shown in Fig. 6. The traction field is applied on the discrete fluid interface $\Gamma_{\mathrm{f}}^{h}$, and piecewise linear basis functions are defined with respect to discrete solid interface $\Gamma_{\mathrm{f}}^{h}$. For this simple geometry, the analytical solution for piecewise continuous traction fields is known along $\Gamma$.

Let us denote by $\boldsymbol{x}_{\mathrm{f}}^{i}, i=1, \ldots m_{\mathrm{s}}$, the nodes on $\Gamma_{\mathrm{f}}^{h}$. We associate with each node $\boldsymbol{x}_{\mathrm{f}}^{i}$ the corresponding unit normal vector $\boldsymbol{n}_{\mathrm{f}}^{i}$ and the piecewise linear basis function $N_{\mathrm{f}}^{i}$. In the model problem, the fluid traction $\boldsymbol{t}_{\mathrm{f}}$ is simply

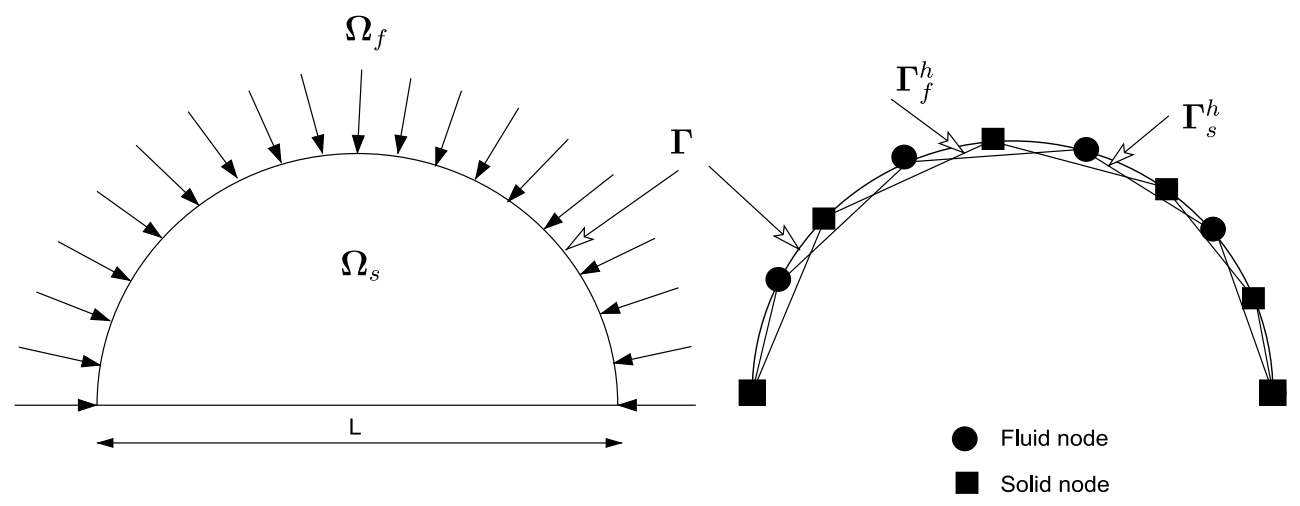

Fig. 6. Circular-arc problem. Left: geometry; Right: interface discretization. 
given by $\boldsymbol{t}_{\mathrm{f}}=p \boldsymbol{n}_{\mathrm{f}}$, where $p$ is assumed to be uniform for simplicity. The approximation $\tilde{\boldsymbol{n}}_{\mathrm{f}}$ for the fluid normal $\boldsymbol{n}_{\mathrm{f}}$ is given by $\tilde{\boldsymbol{n}}_{\mathrm{f}}=\sum_{i=1}^{m_{\mathrm{f}}} N_{\mathrm{f}}^{j} \boldsymbol{n}_{\mathrm{f}}^{j}$. From (6) and (8), we then have

$$
\tilde{\boldsymbol{n}}_{\mathrm{s}}^{i}=-\left[\boldsymbol{M}_{\mathrm{s}}^{i k}\right]^{-1}=\sum_{k=1}^{m_{\mathrm{s}}} \boldsymbol{n}_{\mathrm{f}}^{j} \int_{\Gamma}=N_{\mathrm{f}}^{i} N_{\mathrm{s}}^{k} \mathrm{~d} \Gamma
$$

where $\left[\boldsymbol{M}_{\mathrm{s}}^{i k}\right]=\int_{\Gamma} N_{\mathrm{s}}^{i} N_{\mathrm{s}}^{k} \mathrm{~d} \Gamma$. Due to the non-matching nature of the meshes, there is a regularity problem of numerical quadrature arises when evaluating the integral

$$
\int_{\Gamma} N_{\mathrm{f}}^{j} N_{\mathrm{s}}^{k} \mathrm{~d} \Gamma
$$

The Gaussian or Newton-Cotes quadrature rule assumes smoothness of the integrand functions within an interval and they can be arbitrary inaccurate for non-smooth functions [21].

The basic objective is thus to analyze the transfer of the discretized normal vector field, $\boldsymbol{n}$, onto the solid side discrete interface $\Gamma_{\mathrm{s}}^{h}$ using (45). Note that this normal vector transfer procedure needs an inversion of the consistent mass matrix to compute the projected normal (traction) field on the solid side. However, this linear inversion of the mass matrix may be avoided in the transient fluid-solid interaction problems as the solid solver utilizes the external load vector from (8). In the next three subsections, various aspects of the normal vector transfer are analyzed.

\subsection{Asymptotic analysis}

To explain the different behavior of the discretized normal vector transfer, we consider representative fineto-coarse $\left(h_{\mathrm{s}} / h_{\mathrm{f}}=1.5\right)$ and coarse-to-fine $\left(h_{\mathrm{s}} / h_{\mathrm{f}}=0.75\right)$ discretizations over $\Gamma_{\mathrm{f}}^{h}$ and $\Gamma_{\mathrm{s}}^{h}$. The shape functions are assumed to be linear, so the approximation of the normals on $\Gamma_{\mathrm{f}}$ is piecewise linear. The analytical input normals for the fine-to-coarse projection are shown in Fig. 7(a).

In the fine-to-coarse projection, we see that both the source- and target-based common-refinement schemes project the normals matching with the analytical normal vector field (Fig. 7(b)). Due to the lack of regularity, the point-to-element projection schemes show large errors in the transfer of normals in terms of both direction and magnitude (Fig. 7(c)-(d)). Further, in the case of coarse-to-fine projection (Fig. 7(e)-(f)), the target-based common-refinement scheme again yields normal vectors similar to the analytical ones, while the nodeprojection scheme yields inaccurate transfer of the discretized normals, and in some cases reversed the normal direction due to inaccurate projection.

Let us introduce the relative error in the normal vector $\boldsymbol{n}_{\mathrm{s}}$, as

$$
\epsilon_{\mathrm{n}}=\frac{\left\|\boldsymbol{n}_{\mathrm{s}}-\boldsymbol{n}_{\mathrm{s}}^{\text {exact }}\right\|_{2}}{\left\|\boldsymbol{n}_{\mathrm{s}}^{\text {exact }}\right\|_{2}},
$$

where $\boldsymbol{n}_{\mathrm{s}}^{\text {exact }}$ is computed over $\Gamma_{\mathrm{s}}$ from the exact normal field, and $\boldsymbol{n}_{\mathrm{s}}$ is obtained from (45). Using a Taylor series expansion, the asymptotic error of non-conservative consistent interpolation method [8] across the non-matching interface is $\mathrm{O}\left(h_{\mathrm{f}}^{2}+h_{\mathrm{s}}^{2}\right)$ for a given piecewise continuous normal vector field. However, due to inaccurate transfer, the point-to-element projection schemes are sub-optimal and the local errors along the interface do not necessarily diminish with mesh refinement. The common-refinement methods rely on the weighted residual method, which provides optimal and conservative projection of the normal field in the $L_{2}$ space defined by the solid shape functions. Therefore, the asymptotic errors of such methods have the same order of accuracy as the consistent interpolation method [14], as shown in Fig. 8(a). The ratio of the number of target (solid) to source (fluid) edges is kept constant at 1.25. The error measured in the $L_{2}$ norm is calculated at each refinement step from the known analytical normal (traction) vector field and the varying mesh size $h_{\mathrm{s}}$ is multiplied by the curvature $\kappa$. As apparent in Fig. 8(a), the source-based common-refinement has optimal convergence (i.e., quadratic convergence) with the geometric interpolation of $\mathrm{O}\left(h_{\mathrm{f}}^{2}+h_{\mathrm{s}}^{2}\right)$ while the target-based common refinement enables exact transfer of the uniform normals. As expected, we can see sub-optimal behavior of the point-to-element projection schemes, which have order of accuracy $\mathrm{O}\left(h^{q}\right)$, where $q$ is less than one. 
(a)

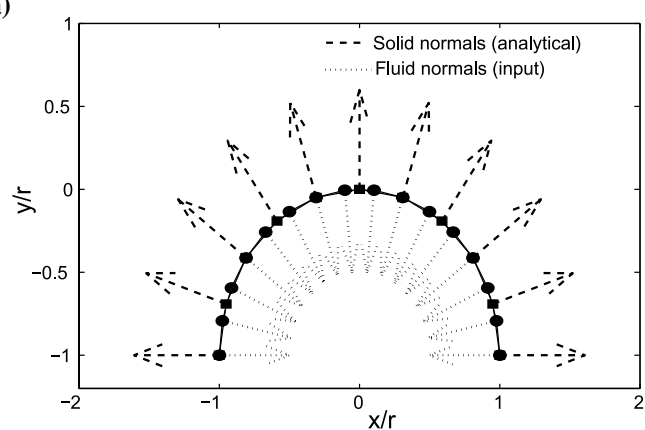

(c)

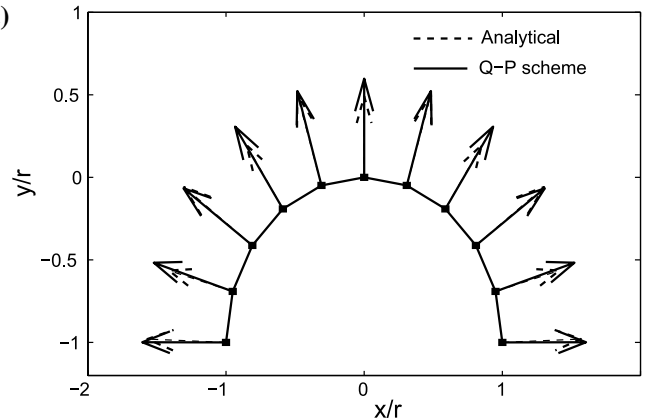

(e)

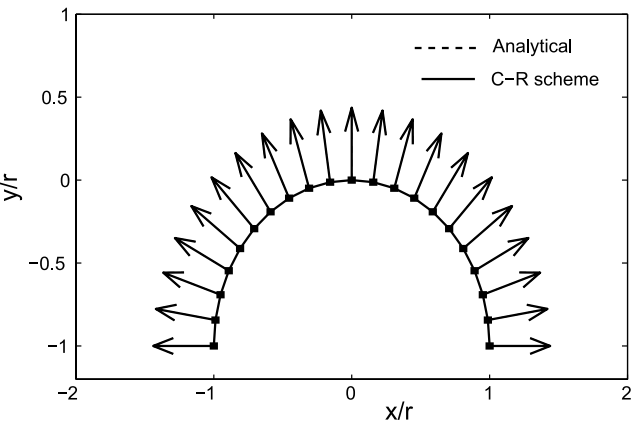

(b)
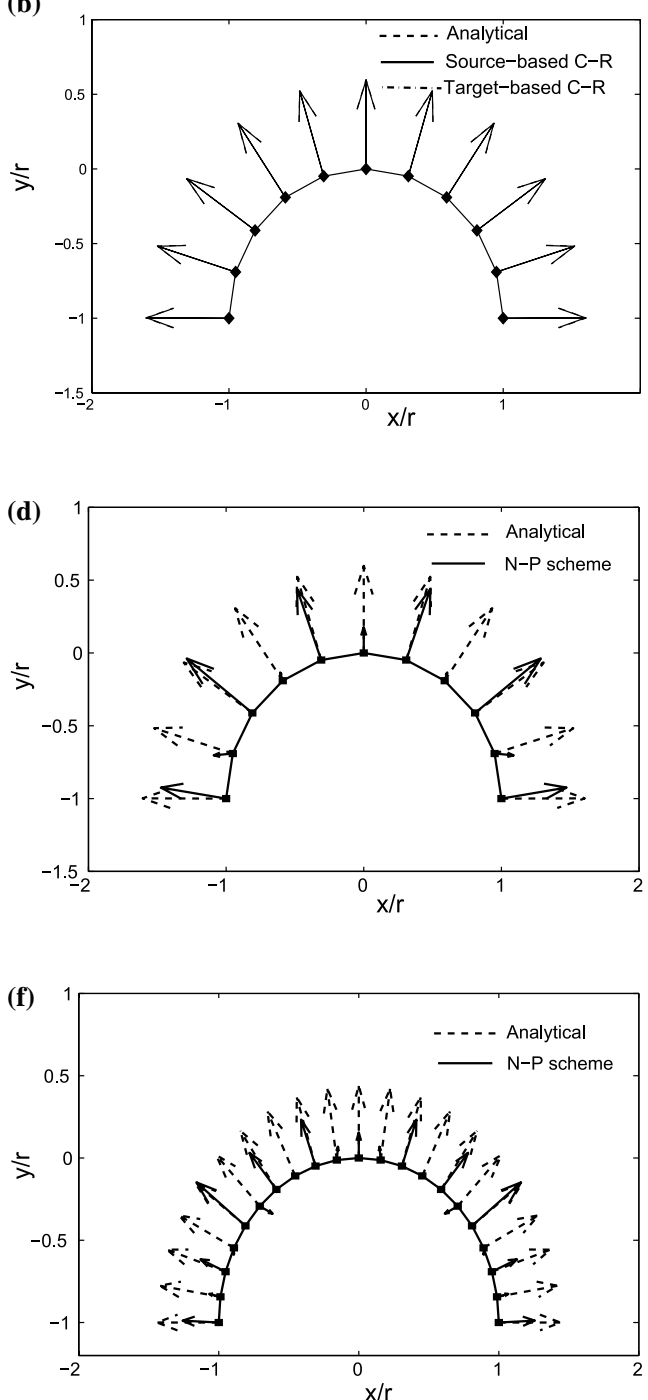

Fig. 7. Transfer of discretized normals onto discrete solid interface: (a)-(d) Fine-to-coarse projection with $h_{\mathrm{s}} / h_{\mathrm{f}}=1.5:(\mathrm{a})$ input normals, (b) common-refinement, (c) quadrature-projection and (d) node-projection. (e)-(f) Coarse-to-fine projection with $h_{\mathrm{s}} / h_{\mathrm{f}}=0.75$ : $(\mathrm{e})$ common-refinement, (f) node-projection.

Next, we analyze the load conservation of common-refinement based methods. To quantify the conservation error, we define $\epsilon_{\mathrm{c}}$ for the source- and target-discretizations on the two meshes and for the reference interface as

$$
\epsilon_{\mathrm{c}}= \begin{cases}\frac{\left\|\int_{\Gamma_{\mathrm{f}}} t_{\mathrm{f}} \mathrm{d} \Gamma-\int_{\Gamma_{\mathrm{s}}} t_{\mathrm{s}} \mathrm{d} \Gamma\right\|_{1}}{\left\|\int_{\Gamma_{\mathrm{f}}} t_{\mathrm{f}} \mathrm{d} \Gamma\right\|_{1}} & \text { on two meshes } \Gamma_{\mathrm{f}} \text { and } \Gamma_{\mathrm{s}}, \\ \frac{\left\|\int_{\Gamma_{\mathrm{r}}}\left(\boldsymbol{t}_{\mathrm{f}}-t_{\mathrm{s}}\right) \mathrm{d} \Gamma\right\|_{1}}{\left\|\int_{\Gamma_{\mathrm{f}}} t_{\mathrm{f}} \mathrm{d} \Gamma\right\|_{1}} & \text { on reference surface } \Gamma_{\mathrm{r}} .\end{cases}
$$

As apparent in Fig. 8(b), the source-based subelement discretization is conservative over two distinct interface discretizations. However, due to differing areas, the conservation error of the target-based subelement 

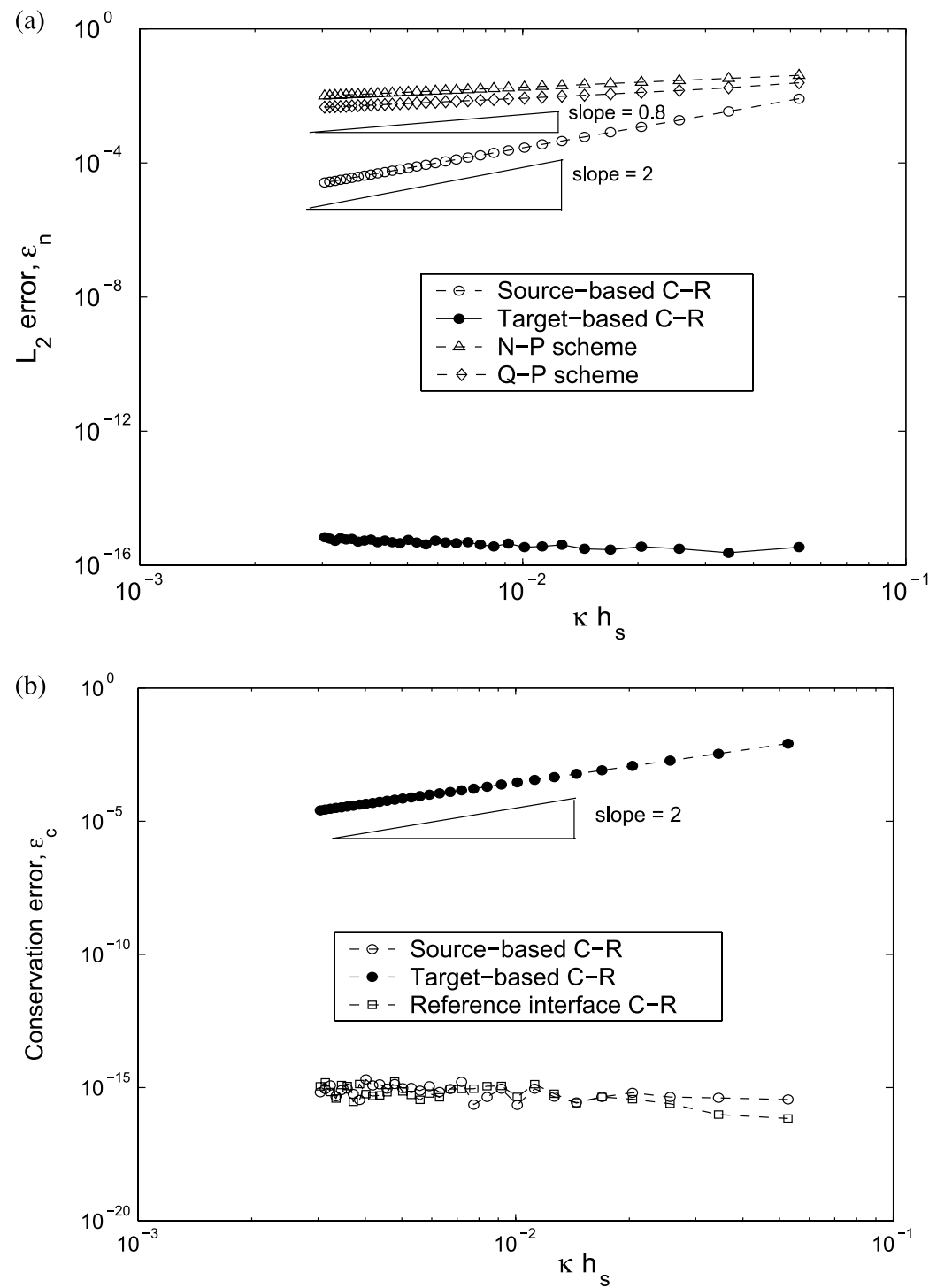

Fig. 8. Decay of the error under simultaneous refinement: (a) accuracy of load transfer schemes in $L_{2}$ norm error $\epsilon_{\mathrm{n}}$ from analytical solution, (b) load conservation error $\epsilon_{\mathrm{c}}$ for two variants of common-refinement and over reference interface $(\alpha=0.5$ and $\beta=0.5)$. $\epsilon_{\mathrm{n}}$ and $\epsilon_{\mathrm{c}}$ are defined in (47) and (48), respectively.

discretization decays with mesh resolutions as $\mathrm{O}\left(h_{\mathrm{f}}^{2}+h_{\mathrm{s}}^{2}\right)$. When a reference unique interface is used, the errors in the load conservation is not affected by the discrepancy in the geometric interpolation. Therefore, we can have an optimal convergence in the accuracy with strict load conservation over the unique reference interface. In the remainder of this paper, we therefore employ only target-based subelements as accuracy is a primary concern and conservation of energy over the reference interface is sufficient for the stability of the coupled simulations as discussed in subsection 3.2.

\subsection{Grid mismatch analysis}

The semi-circular arc problem can also be used to assess the sensitivity and accuracy of the load transfer schemes with respect to mesh resolution and mismatch. A range of grid mismatches is obtained by varying 
the ratio of the numbers of source (fluid) and target (solid) elements (with matching end points). A traction vector profile is prescribed analytically over the interface to have either constant, linear, or quadratic functions on $\Gamma_{\mathrm{f}}^{h}$. The number of nodes of the fine mesh is fixed, while that of the coarse mesh varies. We compute the relative error in the load vector $\mathscr{R}_{\mathrm{s}}$ as

$$
\epsilon_{1}=\frac{\left\|\mathscr{R}_{\mathrm{s}}-\mathscr{R}_{\mathrm{s}}^{\text {exact }}\right\|_{2}}{\left\|\mathscr{R}_{\mathrm{s}}^{\text {exact }}\right\|_{2}},
$$

where $\mathscr{R}_{\mathrm{s}}^{\text {exact }}$ is computed over $\Gamma_{\mathrm{s}}$ from the analytical traction field. If the traction field over $\Gamma_{\mathrm{s}}^{h}$ was obtained by solving (6), then relative errors in the tractions could be defined similarly.

As seen in Fig. 9, large errors in the quadrature-projection scheme compared to that for common-refinement scheme occur due to the lack of regularity of the quadrature rules. To verify this claim and characterize the oscillatory patterns in the errors of the point-to-element schemes for the two uniformly spaced meshes with the size of $h_{1}$ and $h_{2}$, we define a normalized curvature function for the linear basis functions as

$$
\mathrm{CF}\left(h_{1}, h_{2}\right)=\kappa h_{2}\left[\left(\frac{h_{1}}{h_{2}}\right)^{2}+1\right],
$$

and a grid-mismatch function [13]:

$$
\operatorname{GMM}\left(h_{1}, h_{2}\right)=\left|\max \left\{\operatorname{nint}\left(\frac{h_{1}}{h_{2}}\right) \times\left(\frac{h_{2}}{h_{1}}\right),\left(\frac{h_{2}}{h_{1}}\right)\right\}-1\right|,
$$

where $\kappa$ represents the discretized curvature for a given segment of the target mesh and nint function rounds a real number to its nearest integer. The GMM function for a given mesh measures how close the ratio $h_{1} / h_{2}$ is to a positive integer, and a larger value of GMM would, in general, correspond to a more severe violation of the regularity assumption.

Since the common-refinement scheme solves the least squares minimization (3) for non-matching meshes, the overall error of this scheme is directly proportional to the curvature function based on $h_{1}=h_{\mathrm{s}}$ and $h_{2}=h_{\mathrm{f}}$. Therefore, we estimate the common-refinement error as

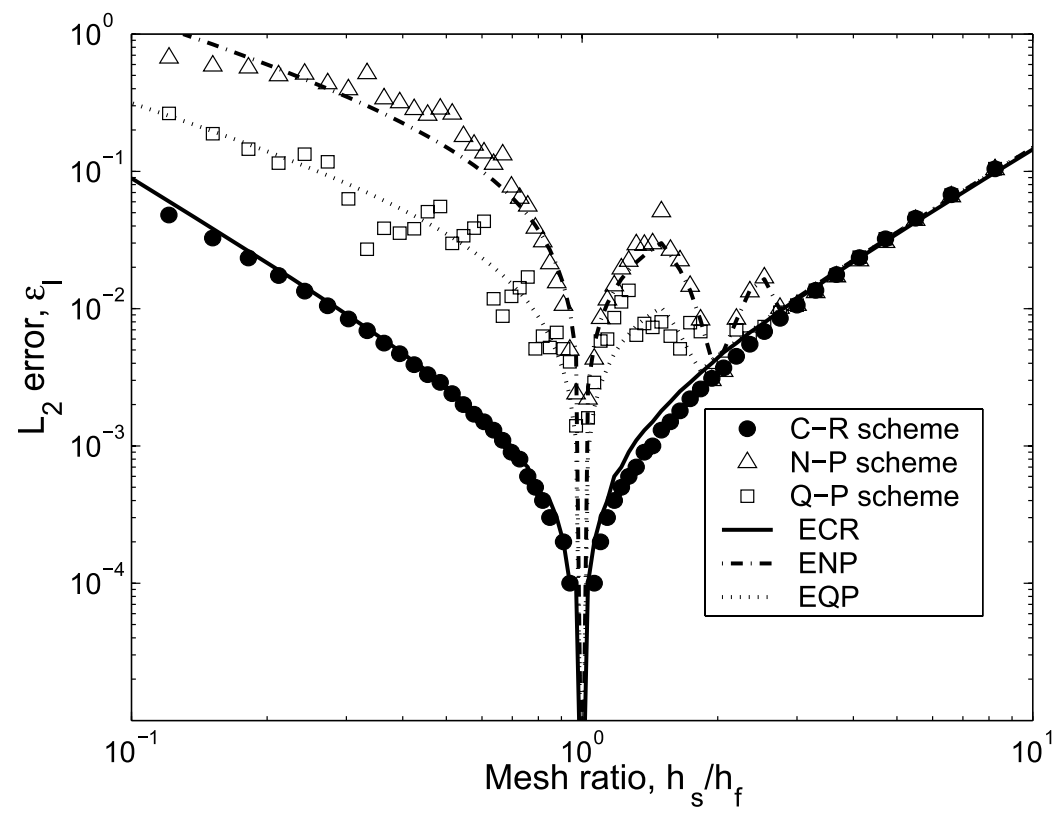

Fig. 9. Dependence of load vector error $\epsilon_{1}$ defined in (49) on relative mesh ratio $h_{\mathrm{s}} / h_{\mathrm{f}}$ for the three load transfer schemes, and correlation with the corresponding analytical error functions in (52)-(54) with $a=0.015$ and $b=0.96$. 


$$
\mathrm{ECR}=a \mathrm{CF}\left(h_{\mathrm{s}}, h_{\mathrm{f}}\right),
$$

where $a$ is a constant, which can be adjusted to correlate well with the overall numerical error. For the nodeprojection (N-P) scheme, the total error includes grid mismatch and thus takes the form:

$\mathrm{ENP}=\max \left[a \mathrm{CF}\left(h_{\mathrm{s}}, h_{\mathrm{f}}\right), b \mathrm{GMM}\left(h_{\mathrm{s}}, h_{\mathrm{f}}\right)\right]$
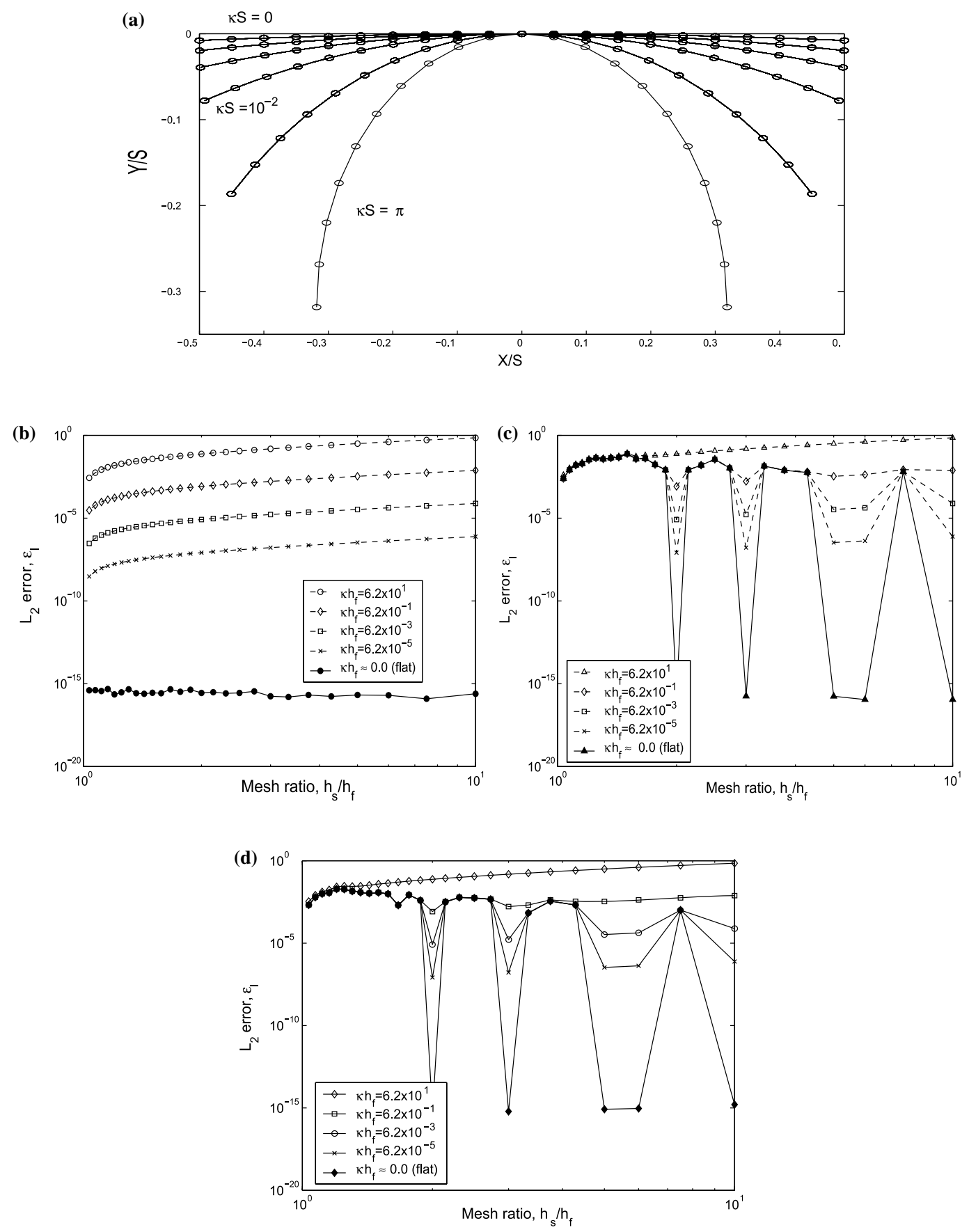

Fig. 10. Effect of curvature: (a) circular arcs with varying curvature, and $L_{2}$ errors in load transfer: (b) common-refinement, (c) nodeprojection and (d) quadrature-projection. $S$ is the fixed perimeter of the circular arcs and $h_{\mathrm{f}}$ is the fixed fluid mesh size. 


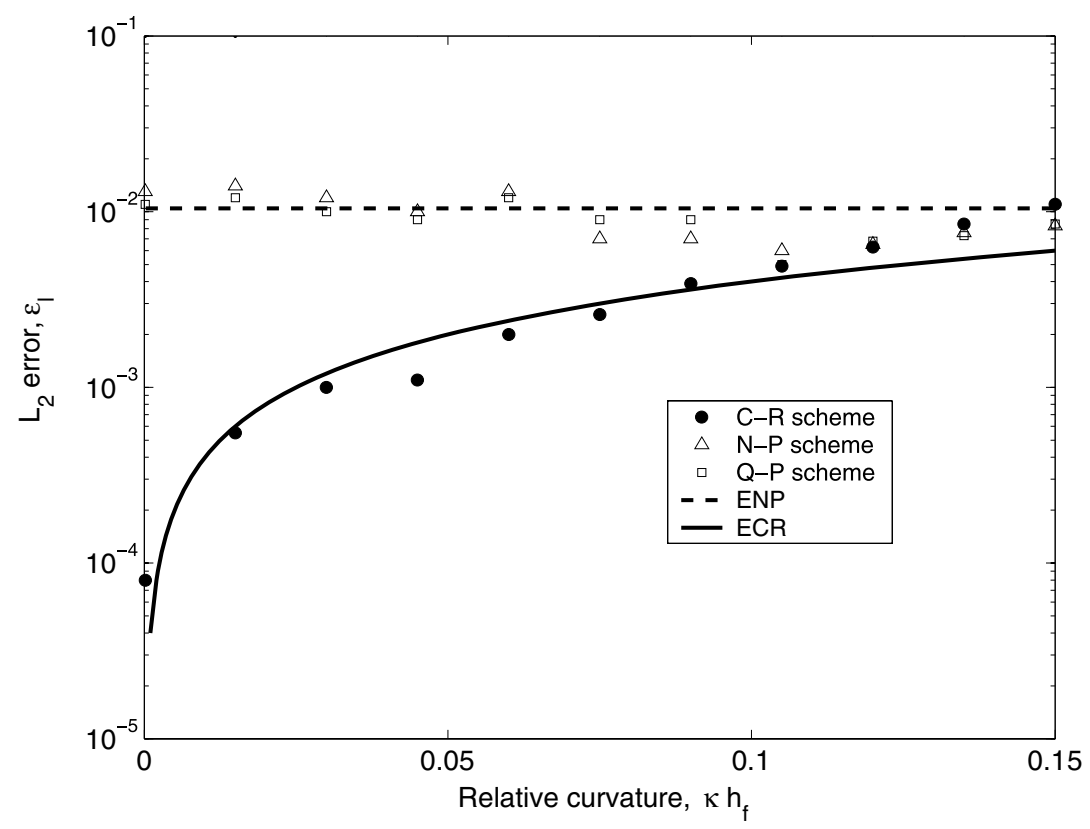

Fig. 11. Effect of the curvature $\kappa$ on the least squares error associated with the three load transfer schemes at various curvatures $\kappa$ for a mesh ratio $h_{\mathrm{s}} / h_{\mathrm{f}}=1.18$ and expected correlation with the corresponding analytical error functions.

as demonstrated in Fig. 9, where $b$ is another constant. For the quadrature-projection (Q-P) scheme, which applies an adaptive quadrature rule when the fluid (source) mesh is coarse, the errors can be expected to correlate with the symmetric grid-mismatch function,

$$
\mathrm{EQP}=\max \left[a \operatorname{CF}\left(h_{\mathrm{s}}, h_{\mathrm{f}}\right), b\left(\min \left\{\operatorname{GMM}\left(h_{\mathrm{s}}, h_{\mathrm{f}}\right), \operatorname{GMM}\left(h_{\mathrm{f}}, h_{\mathrm{s}}\right)\right\}\right)\right] .
$$

As can be seen in Fig. 9, the common-refinement scheme performs within the interpolant error for all the cases. For the node- and quadrature-projection schemes, however, the $L_{2}$ error depends strongly on the grid mismatch. The errors in these two schemes oscillate with the grid mismatch and match with the interpolant error at isolated points where the fine mesh is nested in the coarse mesh (e.g., $h_{\mathrm{s}} / h_{\mathrm{f}}=2.0$ ). Therefore, these above error expressions in (52)-(54) reasonably capture the errors for all the three schemes and can be useful for a priori accuracy assessment. In particular, this key result will allow extension of this work to adaptive interfacing methods.

\subsection{Effects of curvature}

We now study the effect of curvature on the load transfer schemes using the set of circular arcs shown in Fig. 10(a). Fig. 10(b)-(d) present $L_{2}$ error with respect to the mesh ratio $h_{\mathrm{s}} / h_{\mathrm{f}}$ for the three schemes with varying curvatures. It is apparent that the error in the common-refinement based scheme decreases monotonically as the curvature decreases. At $\kappa \approx 0.0$, this load transfer scheme reduces to the flat interface solution [13] and produces exact load transfer within machine precision. On the other hand, the errors in the point-to-element projection schemes, which can be approximated by ENP in (53), do not decrease monotonically as the curvature decreases, and the errors depend on the grid mismatch. These trends are demonstrated in Fig. 11, which shows the $L_{2}$ error with respect to the curvature values at a representative mesh ratio, $h_{\mathrm{s}} / h_{\mathrm{f}}=1.18$.

\section{Superseismic shock-bump problem}

We now turn our attention to an actual transient FSI problem consisting of a shock in a compressible fluid traveling at a superseismic speed over a curved elastic half space, i.e., at a speed $V_{s h}$ that exceeds the 
dilatational wave speed of the solid. This problem presents an interesting coupling between fluid and solid solutions since the deformation of the elastic solid behind the traveling shock affects the fluid flow by changing the shock angle and thereby the intensity of the pressure jump across the shock. Although difficult to produce physically, this coupled problem is studied here because it has an analytical self-similar solution (in the frame of the traveling shock) for the special case of a flat interface and has been well studied [13]. The curved boundary case studied here does not have an analytical solution but still constitutes an excellent test problem to quantify the effect of the curvature on the precision of the load transfer along the fluid-solid interface.

The problem geometry and its discretization with regular and uniform triangles are illustrated in Fig. 12 for the representative case $\kappa L=1.8$, where $\kappa$ and $L$ are the curvature and horizontal length of the bump, respectively. A close-up of the gaps between the non-matching meshes along the bump is also shown in the figure. In this study, we select the fluid flow and solid material properties such that the acoustic impedances of the two media are comparable and a significant two-way coupling occurs in a very short time duration. For the linear elastic solid, we use the properties similar to those of copper: Poisson's ratio $v=0.33$, density $\rho_{\mathrm{s}}=8970 \mathrm{~kg} / \mathrm{m}^{3}$ (to ensure superseismic conditions), and Young's modulus $E=110 \mathrm{GPa}$. For the fluid, we use a perfect gas with the ratio of the specific heats, $\gamma=1.4$, an artificially high initial density, $\rho_{1}=1033 \mathrm{~kg} / \mathrm{m}^{3}$, and an initial pressure $p_{1}=5.55 \mathrm{GPa}$. The initial upstream Mach number for this problem is $M_{1}=2.952$. We consider the unshocked fluid system to be under initial pressure $p_{1}$ and the solid domain to be traction free, i.e., there is a zero applied pressure load in the undisturbed fluid region.

\subsection{Grid mismatch analysis}

Fig. 13(b)-(d) present contour plots obtained with the three load transfer schemes using the same spatial and temporal discretization for a curved non-matching interface with $h_{\mathrm{s}} / h_{\mathrm{f}}=1.18$ at $\kappa L=0.75$. Fig. 13(b) corresponds to the common-refinement based load transfer scheme. For this load transfer scheme, the fluid and solid solutions are quite uniform and very similar to matching interface solutions. On the other hand, the results shown in Fig. 13(c) and (d) exhibit significant spurious oscillations in the shear stress along the curved interface due to the inaccurate load transfer by the Q-P and N-P schemes, respectively, as also found in [13] for flat interfaces.

This effect is further quantified in Fig. 14(a), which shows the normalized shear stress distribution along the curved bump boundary for $h_{\mathrm{s}} / h_{\mathrm{f}}=1.18$ at $t=80 \mathrm{~ms}$. As compared to the matching solution, the point-toelement projection schemes yield an oscillatory solution behind the shock obtained with errors reaching up

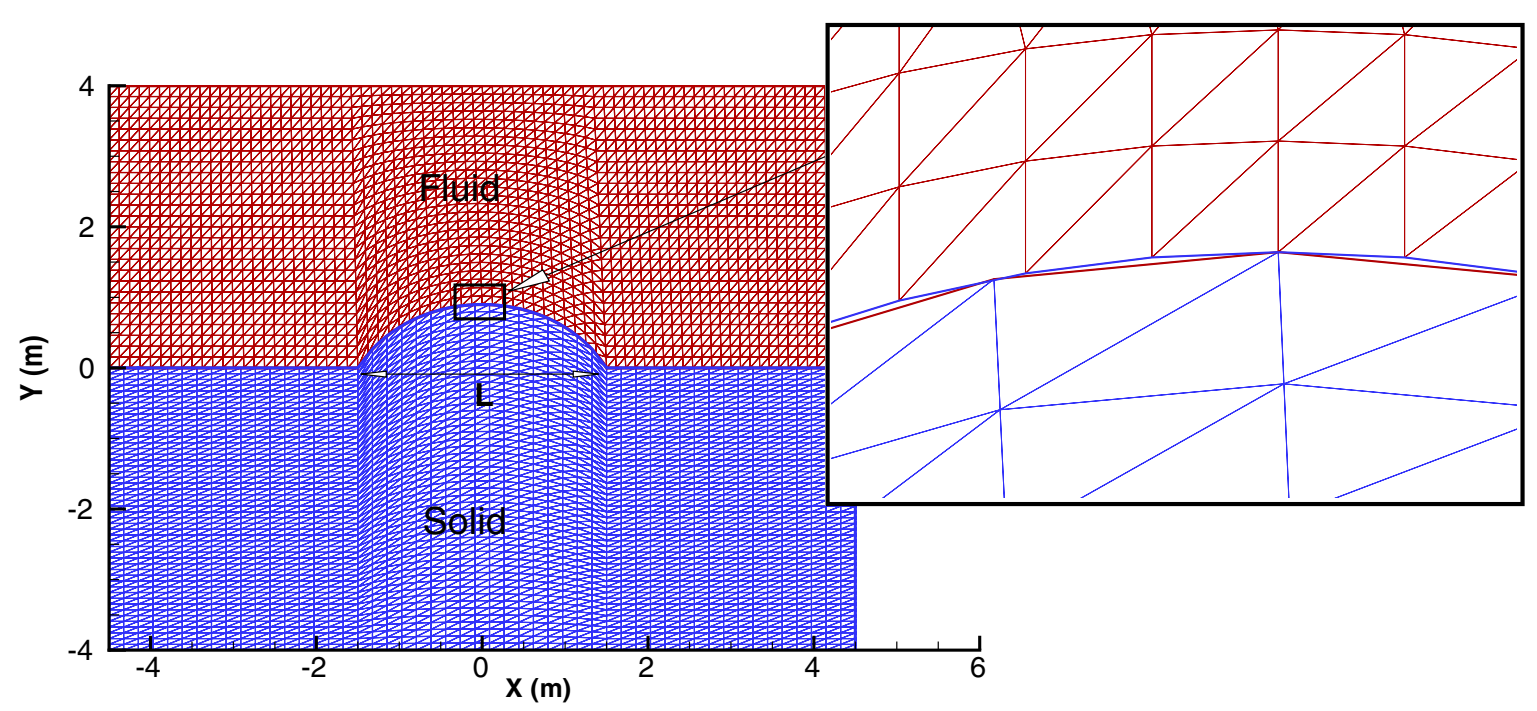

Fig. 12. Superseismic shock-bump problem: representative non-matching meshes with grid ratio of $h_{\mathrm{s}} / h_{\mathrm{f}}=2.22$ at $\kappa h_{\mathrm{f}}=0.09(\kappa L=1.8)$. 
(a)

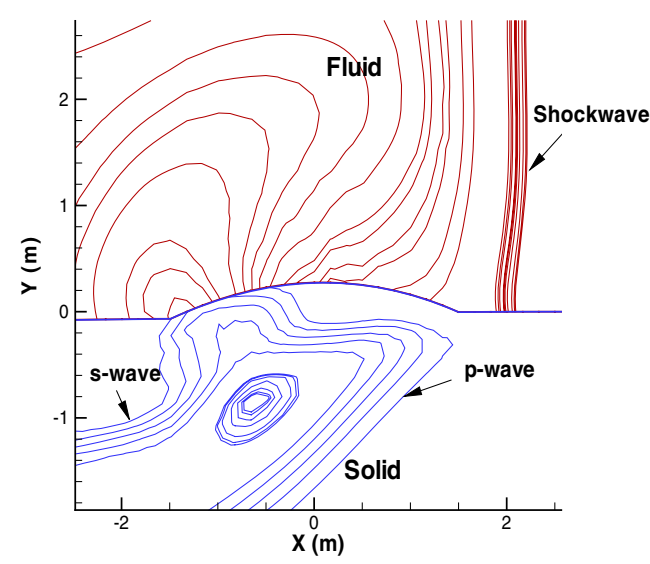

(c)

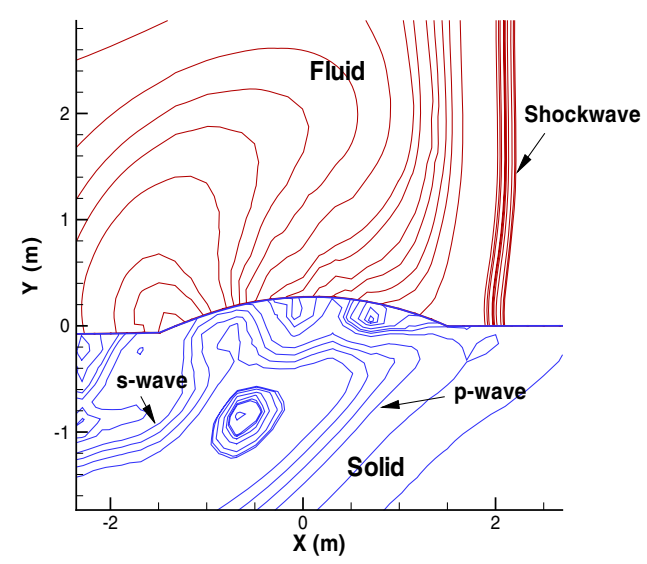

(b)

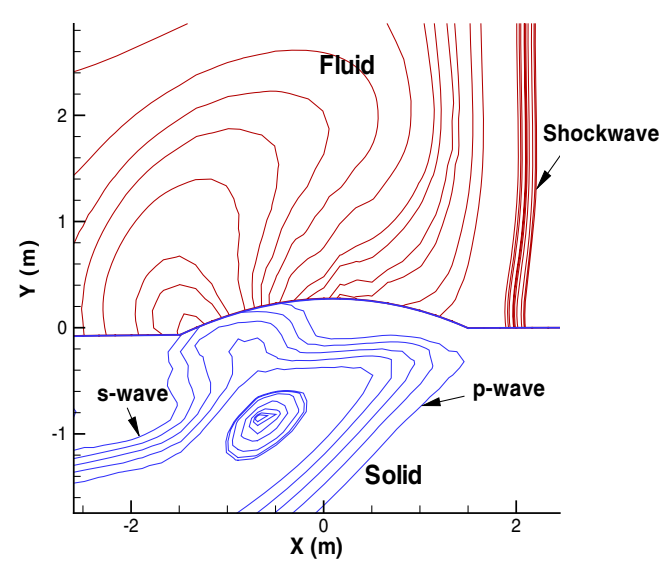

(d)

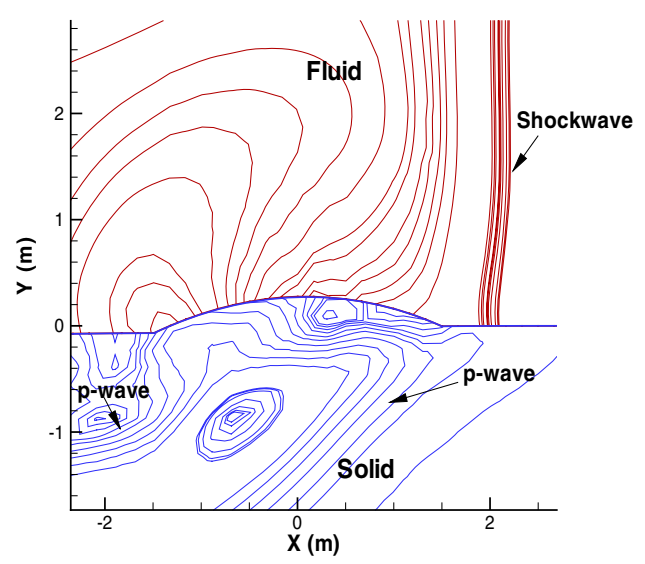

Fig. 13. Superseismic shock-bump problem with $\kappa L=0.75$ : contours of pressure in the fluid domain and shear stress in the solid domain for various load transfer schemes: (a) matching meshes $\left(h_{\mathrm{s}} / h_{\mathrm{f}}=1.0\right)$, (b) common-refinement based scheme, (c) quadrature-projection scheme, (d) node-projection scheme. In (b), (c), and (d), the interface meshes do not match and $h_{\mathrm{s}} / h_{\mathrm{f}}=1.18$.

to $5 \%$ in the normalized shear stress distribution. The common-refinement scheme, however, yields results almost identical to those obtained with matching meshes (though a higher value of $a$ results due to the increased flow complexity and the introduction of deforming interface). This shows that satisfying the traction continuity locally is critical for the load transfer to prevent spurious oscillations along the interface.

The effect of the grid mismatch on the least squares error on the shear stress along the curved boundary is presented in Fig. 14(b). In the absence of a closed-form analytical solution, the error is computed by comparison with the reference solution with matching meshes. We also see correlations between the error functions and the non-uniformity errors for the point-to-element projection schemes. These results are in agreement with the grid mismatch study of the static circular-arc analytical results presented in the previous section, and highlight the importance of the accurate load transfer across non-matching meshes for fluid-solid interaction applications.

\subsection{Effects of curvature}

As in the static circular-arc problem, we now study the effect of curvature on the solutions for the three load transfer schemes in the superseismic shock-bump problem. The outcome of this study is presented in Fig. 15 in terms of the least squares error on the interface shear stress computed along the curved bump for various 

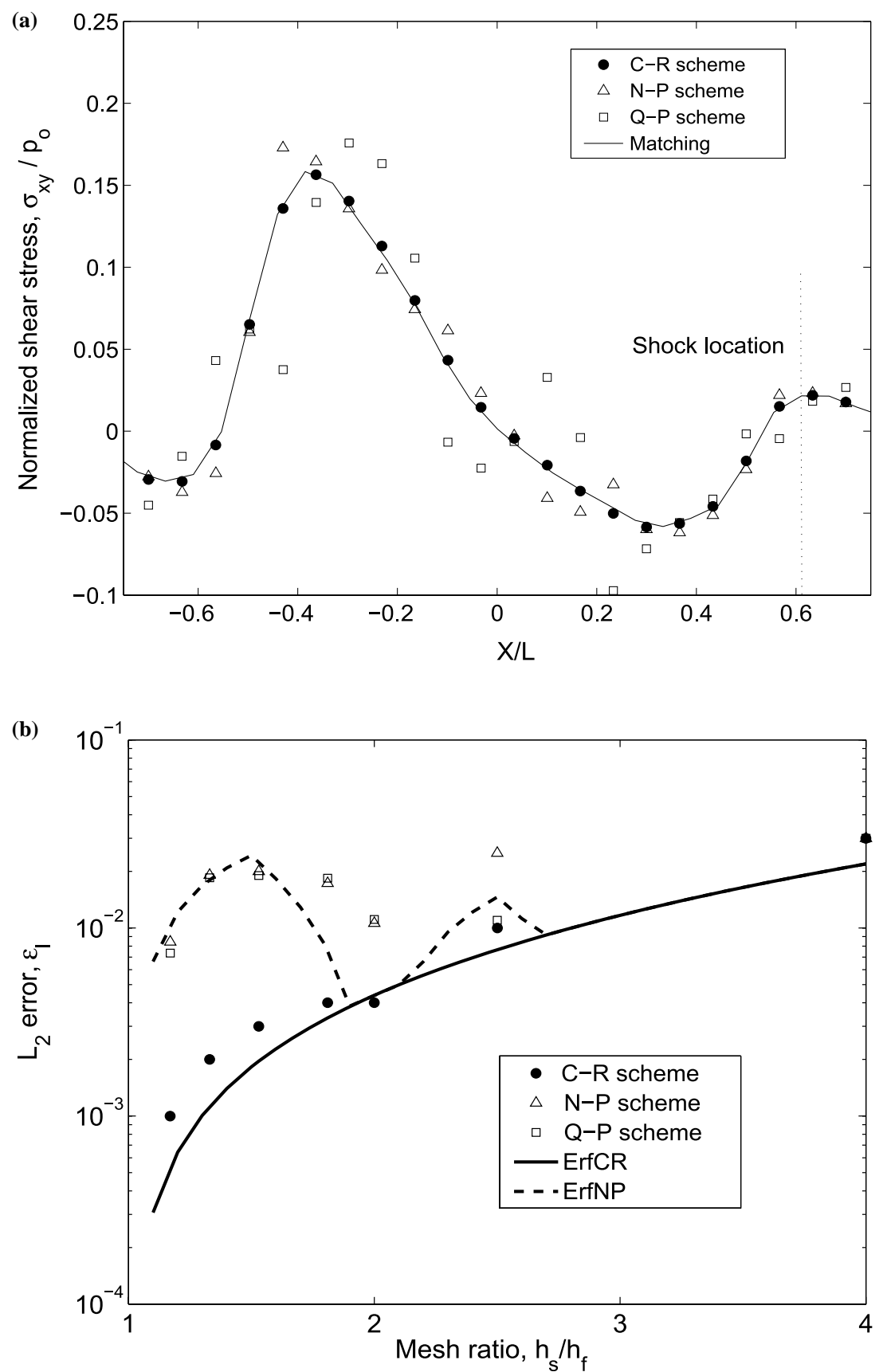

Fig. 14. Solutions along the curved interface $(\kappa L=0.75)$ for three load transfer schemes on non-matching meshes $\left(h_{\mathrm{s}} / h_{\mathrm{f}}=1.18\right)$ and matching reference solution $\left(h_{\mathrm{s}} / h_{\mathrm{f}}=1.0\right)$ at time $t=80 \mathrm{~ms}$ : (a) solid shear stress distribution over $\Gamma_{\mathrm{s}}^{h}$, (b) effect of the grid mismatch on the $L_{2}$ error on the interface shear stress and comparison with the proposed analytical error functions in (14)-(16) with new constant values $a=0.11$ and $b=1.08$.

curvatures for non-matching meshes with $h_{\mathrm{s}} / h_{\mathrm{f}}=1.18$. This figure shows that the $L_{2}$ error in the commonrefinement scheme increases monotonically with the curvature, and the error is minimum for the flat interface case. In contrast, the point-to-element projection schemes show the largest errors for flat interfaces. These findings are consistent with the static circular-arc results (see Fig. 11). 


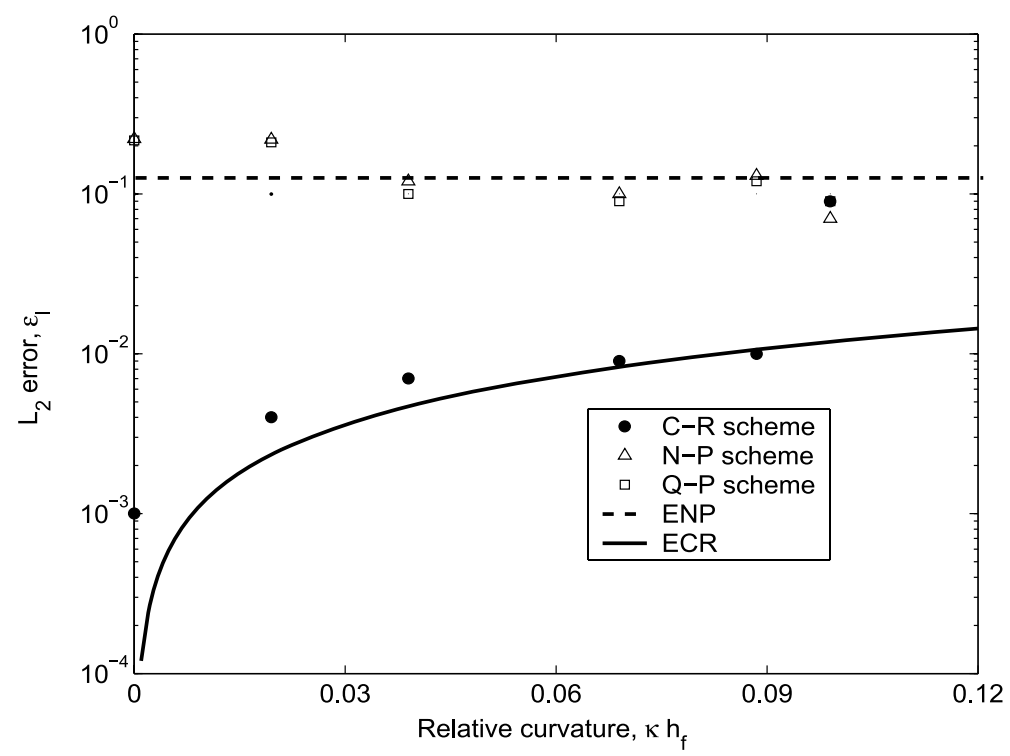

Fig. 15. Least squares error in the interface shear stress associated with the three load transfer schemes for six curvatures values $\kappa$ at $h_{\mathrm{s}} / h_{\mathrm{f}}=1.18$, and expected correlations with the corresponding analytical error functions.

\section{Blast wave problem}

To demonstrate the effects of load transfer schemes on a more practical problem involving a curved interface, we now consider the deformation of a curved solid interface subjected to a blast wave. This study enables accurate assessment of structural strength and failure under sudden blast loading in a safety related situation e.g., solid rocket motor, explosives, chemical processing. A blast wave arises due to the rapid release of energy in a gas. A typical pressure-time curve for an explosive blast wave is presented in [22].

In the present study, the blast wave is assumed to be planar. To generate the blast wave, a high pressure layer of a finite thickness $2 x_{o}$ is considered at a distance $x_{b}$ This problem is similar to the problem of sudden rupture of a membrane in ideal shock tube [23]. After the explosion, the planar shock wave, contact discontinuity and fan of rarefaction waves form and the interaction between the head of rarefaction wave and the shock wave front leads to the nonlinear blast wave profile.

(a)

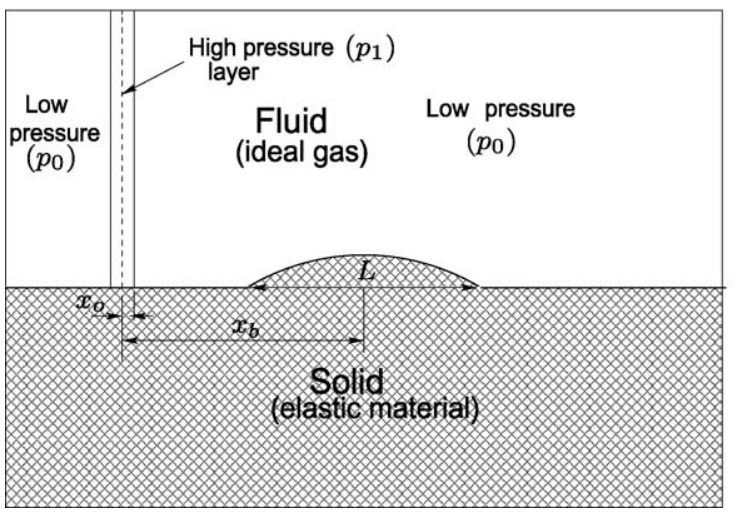

(b)

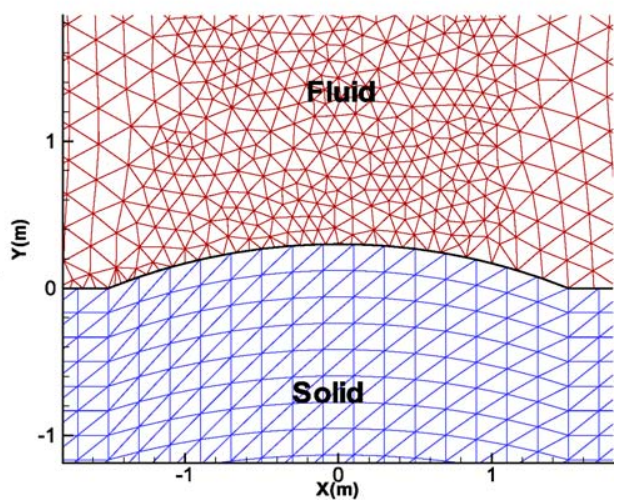

Fig. 16. Blast wave problem: (a) schematic and computational domain, (b) non-matching meshes along the curved boundary with nonuniform spacing of fluid grid points. 
The computational domain with the initial conditions of the blast wave problem is shown in Fig. 16(a). In this representative curved interface problem, $\kappa L$ is equal to 1.8 , where $\kappa$ and $L$ are the curvature and horizontal length of the bump. The high pressure layer is positioned at $x_{b}=1.1 \mathrm{~L}$ and the half-thickness of the layer is $x_{0}=0.033 \mathrm{~L}$. In the solid domain, we use isotropic properties similar to those of the solid propellant: Poisson's ratio $v=0.499$, density $\rho_{\mathrm{s}}=1300 \mathrm{~kg} / \mathrm{m}^{3}$, and Young's modulus $E=10 \mathrm{MPa}$. For the fluid, we use a perfect gas with the physical parameters, an initial density $\rho_{0}=1.293 \mathrm{~kg} / \mathrm{m}^{3}$, and an initial pressure $p_{0}=1.0 \mathrm{~atm}$. The ratio of specific heats $\gamma$ is taken to be 1.4 and the viscosity is neglected in this calculation. The initial parameters in the high pressure layer $p_{1}, \rho_{1}$, etc. are calculated according to the shock tube theory [23]. The initial pressure ratio $p_{1} / p_{0}$ has been set to 33.81 as in [22]. Fig. 16(b) shows the meshes in the fluid and solid domains.
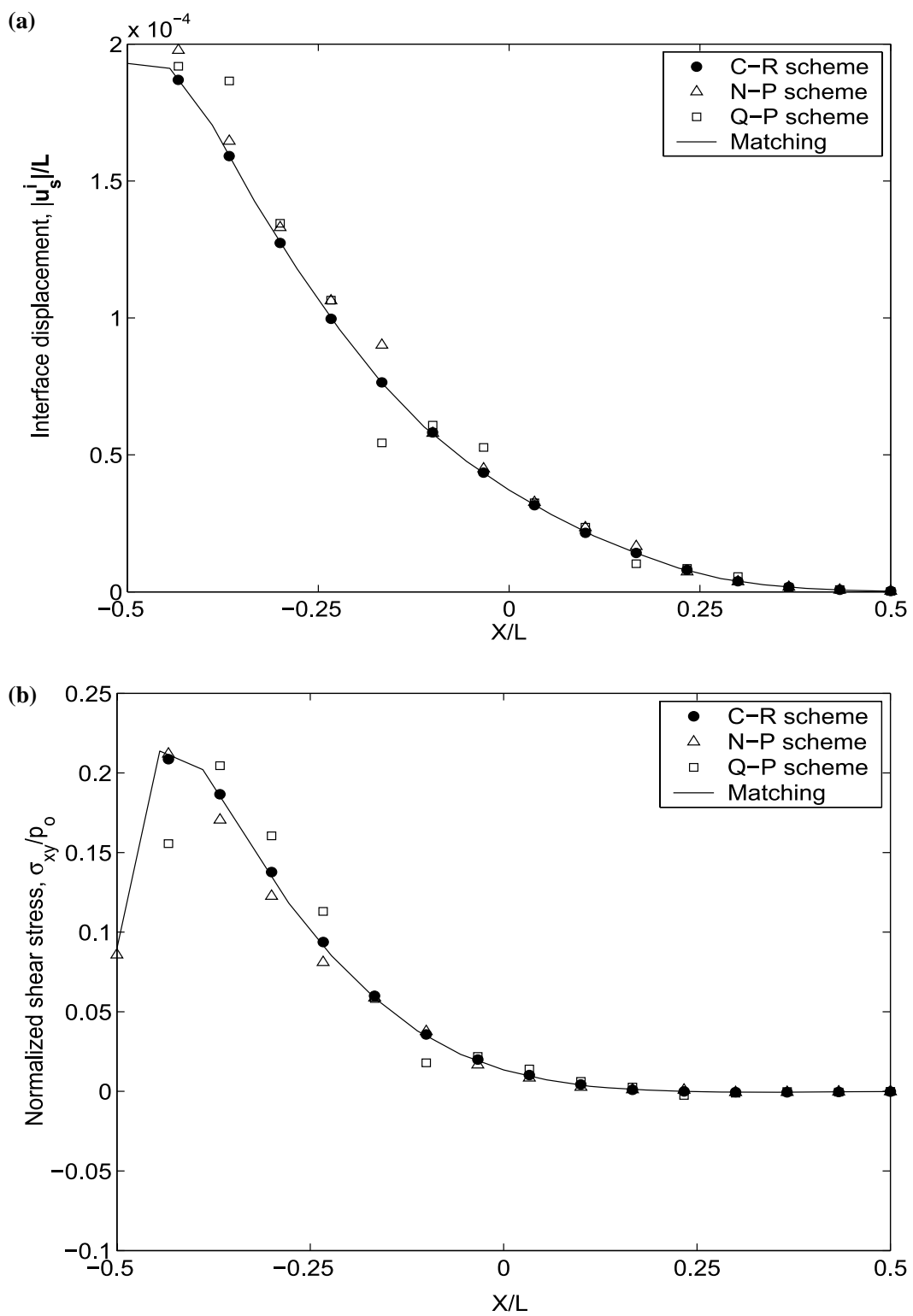

Fig. 17. Blast wave problem: comparison of interface solutions for three load transfer schemes with reference matching solution at time $t=0.2 \mathrm{~s}$ : (a) total displacement magnitude of solid side interface nodes, $\boldsymbol{u}_{\mathrm{s}}^{i}$, normalized by bump length $L$, (b) interface shear stress distribution $\sigma_{x y}$ normalized by initial pressure $p_{0}$. 
The fluid domain has a relatively fine and irregular triangular mesh with the non-uniform distribution of mesh points along the curved boundary.

Transient numerical solutions are obtained with matching and non-matching meshes for this coupled blast wave problem. As expected, a nonlinear blast wave profile propagates over the curved solid boundary introducing small deformations. Fig. 17(a) depicts the total displacement magnitude $\left(\left|\boldsymbol{u}_{\mathrm{s}}^{i}\right|\right)$. The figure clearly illustrates the oscillatory nature of the solution obtained with the two traditional load transfer schemes, with errors reaching up to $20 \%$. The common-refinement scheme, however, yields results almost identical to those obtained with the matching meshes. Fig. 17(b) depicts the normalized shear stress distribution $\left(\sigma_{x y} / p_{0}\right)$ along the interface of the solid domain. Similar behaviour is noted with respect to the accuracy of the commonrefinement scheme and the oscillations of the point-to-element schemes.

It should be noted that the spurious oscillations of the point-to-element treatments did not lead to instabilities severe enough to cause the simulations to stop (blow up). This may be due to numerical dissipation occurred in the complex coupled system consisting of the upwinding-based finite-volume scheme in the moving fluid domain and the standard finite-element scheme in the solid domain.

\section{Conclusion}

We have presented a systematic analysis of conservative load transfer schemes based on point-to-element projection and common-refinement schemes with special emphasis on the effects of grid mismatch and curvature. The common-refinement based projection with a new definition of load conservation over a reference surface has been discussed. By analyzing a general linear fluid-solid interaction model, we have shown that the common-refinement scheme is sufficient for the stability of the coupled simulations while enforcing energy conservation over the reference surface. We have analyzed computations of normals and areas for conservative load transfer over the curved interface boundary and shown that the common-refinement scheme with target-based discretization satisfies the interface conditions across non-matching meshes with load conservation over the reference interface. On the other hand, the point-to-element projection schemes, which violate the interface conditions, were found to exhibit spurious numerical oscillations for a superseismic shock-bump and a blast wave FSI problems. The errors of these schemes were shown to depend on the grid mismatch and did not decrease monotonically as the curvature decreased. In addition, a detailed convergence study has been performed to assess the order of accuracy of the schemes. Finally, simple analytical formulations for the error associated with the various schemes were also constructed and shown to correlate well with observed numerical errors.

\section{Acknowledgements}

The authors wish to acknowledge support from the Center for the Simulation of Advanced Rockets (CSAR) [funded by the US Department of Energy] under subcontract B523819 and from the Computational Science and Engineering (CSE) program at University of Illinois. The authors thank anonymous referees for their helpful comments in improving the presentation of this paper.

\section{References}

[1] J.C. Simo, P. Wriggers, R.L. Taylor, A perturbed Lagrangian formulation for the finite element solution of contact problems, Comput. Methods Appl. Mech. Engrg. 50 (1985) 163-180.

[2] N. El-Abbasi, K.-J. Bathe, Stability and patch test performance of contact discretizations and a new solution algorithm, Comput. Struct. 79 (2001) 1473-1486.

[3] B. Flemisch, M.A. Puso, B.I. Wohlmuth, A new dual mortar method for curved interfaces: 2D elasticity, Int. J. Numer. Methods Engrg. 63 (2005) 813-832.

[4] C. Bernardi, Y. Maday, A.T. Patera, A new nonconforming approach to domain decomposition: The mortar element method, in: H. Brezis, J.L. Lions (Eds.), Nonlinear Partial Differential Equations and their applications College de France Seminar Volume XI, 1994, pp. $13-51$.

[5] X.C. Cai, M. Dryja, M. Sarkis, Overlapping nonmatching grid mortar element methods for elliptic problems, SIAM J. Numer. Anal. 36 (1999) 581-606. 
[6] S.-C. Lee, M.N. Vouvakis, J.-F. Lee, A non-overlapping domain decomposition method with non-matching grids for modeling large finite antenna arrays, J. Comput. Phys. 203 (2005) 1-21.

[7] C.A. Felippa, K.C. Park, Staggered transient analysis procedures for coupled mechanical systems: formulation, Comput. Methods Appl. Mech. Engrg. 24 (1980) 61-111.

[8] M.J. Smith, D.H. Hodges, C.E.S. Cesnik, Evaluation of computational algorithms suitable for fluid-structure interactions, J. Aircraft 37 (2000) 282-294.

[9] G.P. Guruswamy, A review of numerical fluids/structures interface methods for computations using high-fidelity equations, Comput. Struct. 80 (2002) 31-41.

[10] C. Farhat, M. Lesoinne, P. LeTallec, Load and motion transfer algorithms for fluid/structure interaction problems with nonmatching discrete interfaces: momentum and energy conservation, optimal discretization and application to aeroelasticity, Comput. Methods Appl. Mech. Engrg. 157 (1998) 95-114.

[11] J.R. Cebral, R. Lohner, Conservative load projection and tracking for fluid-structure problems, AIAA J. 35 (1997) $687-692$.

[12] S.A. Brown, Displacement extrapolations for CFD+CSM aeroelastic analysis, in: AIAA 97-1090 CP, 1997.

[13] R. Jaiman, X. Jiao, X. Geubelle, E. Loth, Assessment of conservative load transfer on fluid-solid interface with nonmatching meshes, Int. J. Numer. Methods Engrg. 64 (2005) 2014-2038.

[14] X. Jiao, M.T. Heath, Common-refinement based data transfer between nonmatching meshes in multiphysics simulations, Int. J. Numer. Methods Engrg. 61 (2004) 2402-2427.

[15] C. Farhat, P. Geuzaine, C. Grandmont, The discrete geometric conservation law and the nonlinear stability of ALE schemes for the solution of flow problems on moving grids, J. Comput. Phys. 174 (2001) 669-694.

[16] O.C. Zienkiewicz, K. Morgan, Finite elements and approximations, Wiley and Sons, 1983.

[17] X. Jiao, M.T. Heath, Overlaying surface meshes, (part 1): Algorithms, Int. J. Comput. Geom. Appl. 14 (2004) $379-402$.

[18] C. Farhat, M. Lesoinne, Two efficient staggered algorithms for the serial and parallel solution of three-dimensional nonlinear transient aeroelastic problems, Comput. Methods Appl. Mech. Engrg. 182 (2000) 499-515.

[19] J. Boujot, Mathematical formulation of fluid-structure interaction problems, Math. Model. Numer. Anal. 21 (1987) 239-260.

[20] X. Feng, Analysis of finite element methods and domain decomposition algorithms for a fluid-solid interaction problem, SIAM J. Numer. Anal. 38 (2000) 1312-1336.

[21] M.T. Heath, Scientific Computing: An Introduction Survey, second ed., MacGraw-Hill, New York, 2002.

[22] D.K. Ofengeim, D. Drikakis, Simulation of blast wave propagation over a cylinder, Shock Waves 7 (1997) $305-317$.

[23] H.W. Liepmann, A. Roshko, Elements of Gas Dynamics, Wiley, New York, 1957. 